\title{
The Critical Condition for Thermal Explosion in an Isoperibolic \\ System.
}

\author{
D. Sánchez-Rodriguez, J. Farjas*, P. Roura. \\ University of Girona, Campus Montilivi, Edif. PII, E17071 Girona, Catalonia, Spain
}

\begin{abstract}
Knowing the conditions for a system to undergo thermal explosion is of utmost importance for many applications. We present a critical condition that accounts for reactant consumption and covers most practical situations, including low activation energy reactions. Our solution applies to cylindrical reactors of any radius to height ratio. In the case of films, it is shown that thermal explosion is virtually impossible. We also introduce a new criterion to define the boundary of thermal runaway based on heat balance. This new definition of criticality allows us to check the accuracy of the nonstationary model to describe the critical condition. The non-stationary model is the base of most approaches.
\end{abstract}

Keywords: Thermal runaway, runaway criterion, ignition condition, volume combustion synthesis, storage hazard, gasless reactions.

*Corresponding author: jordi.farjas@udg.edu, Tel (34)972418490, Fax (34) 972418098 University of Girona, Campus Montilivi, Edif. PII, E17071 Girona, Catalonia, Spain 


\section{Introduction}

Knowledge of the critical condition to predict the occurrence of a thermal explosion is pertinent for a large number of applications. ${ }^{1}$ It is useful when evaluating the chemical risk associated to the thermal stability of compounds or reacting mixtures and in preventing ignition during the storage and transportation of hazardous materials or in chemical reactors, ${ }^{2-7}$ in determining the conditions for pyrotechnic reactions to occur, ${ }^{8-}$ ${ }^{10}$ to determine munitions cook-off temperatures, ${ }^{11}$ in preventing thermal explosion in batteries $^{12,13}$ and, in general, in establishing the ignition condition for chemical engineering processes. ${ }^{14,15}$

Over the last two decades, combustion has become a very versatile route for the synthesis of intermetallic or oxide powders and compacts. ${ }^{16-21}$ Combustion synthesis takes advantage of the heat evolved during the chemical reaction of precursor substances to obtain materials that would otherwise imply high-temperature processing techniques. ${ }^{16}$ Therefore, highly exothermic reactions of high adiabatic temperatures above $1800 \mathrm{~K}$ are needed. ${ }^{16}$ Recently, combustion synthesis of functional oxides from precursor salts (nitrates or metalorganic precursors) has attracted much attention due to the growing interest in these materials. ${ }^{19,22}$ The decomposition of oxide precursor salts may entail much lower adiabatic temperatures, i.e., of only several hundred kelvins. In other words, the range of thermodynamic and kinetic parameters involved in combustion synthesis has expanded considerably. One striking application is the combustion of bimetallic foils used for joining temperature-sensitive or dissimilar materials. ${ }^{23,24}$ Also, a promising new low-temperature fabrication route to synthetize high-performance metal oxide thin-films based on combustion has recently attracted much attention. ${ }^{25}$ In these particular applications, heat dissipation to the substrate is 
very important because it can hinder combustion by avoiding the overheating needed to set a thermal runaway. Consequently, film thickness is a critical parameter that has been analyzed both experimentally ${ }^{24,26}$ and theoretically. ${ }^{27,28}$ Knowledge of the critical condition for combustion is also important for intermetallic reactions and thermites (metal-metal oxides mixtures) that are used in some pyrotechnic devices such as igniters. These reactions evolve at a very high rate without forming a gas phase product. $^{29}$

We will analyze an isoperibolic system, i.e., the sample is placed in a vessel, the walls of which are kept at a constant temperature $T_{f}$. The system undergoes thermal explosion, when reactants are heated to a temperature where the reaction becomes locally unstable. ${ }^{18,19,27,30}$

Frank-Kamenetskii solution $^{31}$ is probably the best known critical condition for thermal explosion and it has been extensively applied to describe combustion in condensed matter. ${ }^{14,32,33}$ In its derivation, reactant consumption is neglected and it is assumed that the activation energy is infinite. As we will see, the first assumption is accurate in some instances where thermal explosion arises at the very early stages of the reaction but for relatively low enthalpy reactions, such as the decomposition of oxide precursor salts, this may be a poor approximation. ${ }^{34,35}$ A realistic model should take into account reactant consumption and finite values of the activation energy. ${ }^{36,37}$ FrankKamenetskii and other authors improved the analysis by taking into account reactant consumption $^{31,34-36,38-43}$ and finite values of the activation energy. ${ }^{36,42}$

Analytical solutions are limited to idealized geometries that allow the system to be reduced to a one-dimensional (1D) model. A common approach to reduce a cylindrical vessel to a $1 \mathrm{D}$ model is to assume a spherical vessel with the same volume ${ }^{6}$ we will show that this is quite a crude approximation. Several authors have numerically 
explored two-dimensional models $(2 \mathrm{D})^{44,45}$ but a general analytical solution for more realistic geometries is still lacking.

In this paper, we explore numerically the accuracy of existing critical conditions for a range of parameters that covers nearly all practical cases. We show that most models provide an accurate prediction for highly exothermic reactions and high activation energies, but they exhibit significant inaccuracies for low enthalpy or low activation energy reactions. We introduce minor modifications to the existing models to overcome these limitations. This result allows us to determine the critical thickness for thermal explosion to occur in a film. This analysis shows that thermal explosion is virtually impossible to achieve for thin-films. We also expand the critical condition to account for a more realistic vessel geometry. As a result of that, we obtain an analytical solution for the sample's critical mass needed for a thermal explosion to occur.

The structure of this paper is as follows. In the second section, we introduce a 2D model that accounts for heat transport by means of diffusion and heat generation due to a thermally activated exothermic reaction. In the third section we compare numerical results against experimental data to assess the ability of the numerical model to describe real situations. In the fourth section we introduce a new definition of criticality and we analyze the accuracy of several runaway critical conditions. Afterwards, we introduce an improved critical condition for 1D and 2D geometries, respectively. The accuracy of these critical conditions is tested against the numerical and experimental data introduced in the third section. The final section is a practical guide to determine whether a thermal runaway will occur or not.

\section{The model}


The classical theory for ignition ${ }^{1,14,31,40,46,47}$ involves heat generated locally by a chemical reaction and heat propagation through the sample. We ignore the effect of reactive gas depletion or the evolution of the system parameters during the reaction. These are reasonable approximations to predict the onset of a thermal runaway but may result in a poor prediction of the evolution of the reaction for those systems whose parameters evolve during the reaction. For instance, in the occurrence of gas depletion, the reaction front may extinguish. ${ }^{48}$ Therefore, our analysis would provide necessary, but not sufficient condition, for a thermal runaway to take place. The model describes heat propagation in a homogeneous medium. It is a reasonable approximation to describe the macroscopic behavior of front propagation provided that the reaction front is wider than the medium inhomogeneities ${ }^{1}$ or if the time of heat exchange in the heterogeneous medium is much shorter than the time of chemical reaction. ${ }^{49} \mathrm{We}$ also overlook heat losses from convection and radiation. Under these assumptions, and for a cylindrical vessel, the system is reduced to a $2 \mathrm{D}$ model where the rate of temperature change has two contributions, a conduction term and a reaction term

$\rho c \frac{\partial T}{\partial t}=\lambda\left[\frac{1}{r} \frac{\partial}{\partial r}\left(r \frac{\partial T}{\partial r}\right)+\frac{\partial^{2} T}{\partial z^{2}}\right]+\rho q \frac{\partial \alpha}{\partial t}$,

where $\rho$ is the density, $q$ is the specific heat of reaction and $\lambda$ is thermal conductivity, $z$ and $r$ are the axial and radial coordinates, respectively (see Fig. 1) and $\alpha(r, z, t)$ is the degree of conversion ( $\alpha=0$ untransformed, $\alpha=1$ totally transformed). We assume that initially the sample is in thermal equilibrium with the furnace walls and that the degree of conversion is zero throughout the sample. The boundary conditions are:

$T(R, z)=T(r, 0)=T_{f},\left.\quad \frac{\partial T}{\partial r}\right|_{r=0}=0,\left.\quad \frac{\partial T}{\partial z}\right|_{z=H}=0$

where $H$ is the sample height, $R$ is the vessel's inner radius and $T_{f}$ is the vessel temperature. The last condition states that the heat flux is null at the top of the sample 
(no convention, no radiation losses). One may think that the latter assumption is a very rough approach to deal with decomposition reactions where a significant amount of heat is lost through the gases evolved. However, when gases evolve, the heat capacity of the sample diminishes. If the gas is thermalized with the sample, the heat evolved is almost completely compensated with the loss of heat capacity, so the final heat balance is negligible with respect to heat losses through the crucible walls. ${ }^{50}$

The set of boundary conditions, Eq. (2), also describes a closed vessel full of reactant provided that $H$ is the vessel's half height (due to symmetry, for a cylindrical vessel the boundary condition $T(2 H, 0)=T_{f}$ is equivalent to $\left.\partial T /\left.\partial z\right|_{z=H}=0\right)$.

As for the reaction, we assume a $n$-order reaction kinetics to account for the reactant consumption. Moreover, the reaction is thermally activated and we suppose an Arrhenius temperature dependence, i.e.:

$\frac{\partial \alpha}{\partial t}=A e^{-E_{A} / R_{G} T}(1-\alpha)^{n}$

where $A$ and $E_{A}$ are the pre-exponential constant and the activation energy of the reaction rate constant, respectively, and $R_{G}$ is the universal gas constant.

This model has been successfully applied to describe combustion in solids, ${ }^{32}$ thermal explosion in munitions ${ }^{11,51}$ and front propagation speed in pyrotechnic systems. ${ }^{8}$ It has also been shown that it provides a reasonable description for those systems whose parameters evolve with temperature and can be used in solid-gas reactions provided that gas exchange is fast enough and that heat transfer through the gases is negligible. ${ }^{52}$

\section{Experimental and numerical results}


To check the ability of the numerical model to describe real processes, we have analyzed the decomposition of a metalorganic precursor in the form of powders: yttrium trifluoroacetate, $\mathrm{Y}\left(\mathrm{CF}_{3} \mathrm{COO}\right)_{3}\left(\mathrm{Y}(\mathrm{TFA})_{3}\right)$. Preparation details are given in. ${ }^{53}$

The reaction course is monitored by thermogravimetry (TG). TG records the mass of a sample, $m(t)$, when it is submitted to a controlled temperature program. The averaged conversion fraction at any time can be easily calculated as: ${ }^{.5}$

$\bar{\alpha}(t)=\frac{m_{i n}-m(t)}{m_{i n}-m_{f i n}}$.

where $m_{\text {in }}$ is the initial sample mass and $m_{f i n}$ is the final mass.

TG analysis was performed with a Mettler Toledo thermobalance (model TGA/DSC1). Samples were placed inside uncovered alumina crucibles. The crucible's internal and external radii are 2.5 and $3 \mathrm{~mm}$, respectively. Inside the furnace a gas flow rate of $50 \mathrm{~mL} / \mathrm{min}$ was controlled by a mass flow meter. High purity nitrogen was used. TG experiments were performed under isothermal conditions at 280 and $290^{\circ} \mathrm{C}$.

The curves of the transformed fraction versus time for different initial sample masses are shown in Fig. 2. One can observe an evolution of the kinetics with the sample mass that is a characteristic feature of overheating due to the heat released by the chemical reaction. ${ }^{28}$ Also, when the mass changes from 35 to $38 \mathrm{mg}$ the curves develop an abrupt step that reveals the occurrence of a thermal runaway. ${ }^{26,28,55,56}$ Conversely, all curves measured at $280^{\circ} \mathrm{C}$ are smooth. Thus, there is a critical temperature and a critical size above which thermal runaway occurs.

The parameters used to simulate the decomposition of $\mathrm{Y}(\mathrm{TFA})_{3}$ are summarized in Table 1 . These parameters have been determined experimentally. ${ }^{28}$ The activation energy and pre-exponential term have been determined from several TG measurements performed at different heating rates by means of isoconversional kinetic methods. ${ }^{54,57-59}$ 
In Fig. 3 we illustrate the calculated $\bar{\alpha}(t)$ for different temperatures and sample masses. The evolution of the kinetics with temperature and sample size is reproduced by the numerical simulations. At $290^{\circ} \mathrm{C}$ we observe that an abrupt step develops in the $41-42$ mg mass range; thus the predicted critical mass is in fair agreement with that determined from the experiments. Also, in agreement with experiments, no thermal runaway is observed at $280^{\circ} \mathrm{C}$.

In contrast, the numerical simulation fails to provide an accurate description of the reaction course. The reason is that we have assumed a single-step first-order reaction model, which is a very rough approximation to the actual decomposition kinetics of $\mathrm{Y}(\mathrm{TFA})_{3}$. So, there is a fair agreement between the critical masses and temperatures determined from experiments and simulations despite the fact that the reaction course depends on the reaction model. This apparent contradiction is solved if we consider that the thermal runaway occurs at the early stages of the transformation; consequently, a first-order reaction model would correctly predict the threshold provided that it describes accurately enough the first stages of the reaction.

Also, it is important to note that isothermal experiments are far from ideal; the furnace does not reach the isotherm instantaneously but it takes more than 10 minutes to fully stabilize the temperature. Since, during this time lapse, the reaction progresses, a compromise has been taken between furnace thermalization and null initial sample transformation: the experiment onset is taken when the furnace temperature is $4^{\circ} \mathrm{C}$ below the programmed one. Though, this strategy is far from compensating the artifacts due to deviations from the programmed temperature. Indeed, near the thermal runaway threshold, the reaction course is very sensitive to temperature fluctuations around the programmed temperature. 
The occurrence of a thermal runaway is controlled by two competing phenomena: the exothermic reaction that tends to increase the local temperature, and heat dissipation that lowers the temperature through heat conduction. ${ }^{1}$ The heat power released by the reaction is given by

$I_{G e n}=\int_{V} \rho q A e^{-E_{A} / R_{G} T}(1-\alpha)^{n} d V$,

where $V$ is the sample's volume. The heat power dissipated is,

$I_{D i s}=\int_{S} \lambda \vec{\nabla} T d \vec{S}$

where the closed surface $S$ is the boundary between the sample and the vessel.

Semenov $^{60,61}$ showed that, in the subcritical parameter region, a stable low temperature state exists where heat generation is compensated by heat dissipation, $I_{\text {Gen }}=$ $I_{\text {Dis. }}$ In homogeneously heated systems, thermal explosion occurs when this low temperature state is unstable, i.e., heat generated by the reaction exceeds the heat removed through the walls of the vessel, $I_{G e n}>I_{D i s}$. Hence, the occurrence of a thermal runaway can be revealed from the value of the ratio between the heat generation and the heat removal rates, $\phi$ :

$\phi \equiv I_{G e n} / I_{D i s}$.

In Fig. 4 we have plotted the evolution of $\phi$ for two different masses. For a sample below the critical mass (22 mg), after a transient period, $\phi$ evolves to 1 , i.e., it reaches the subcritical regime where heat generation is compensated by heat removal. The transient period is due to the build-up of the temperature gradient related to heat removal. Conversely, for a sample above the critical mass $(56 \mathrm{mg})$, after the transient period, the sample experiences an abrupt increase of heat generation that is responsible for the thermal runaway. Eventually, due to the reactant consumption, the heat generated diminishes. Thus, when thermal runaway occurs, the evolution of $\phi$ goes through a local maximum. 
An example of a simulation where thermal runaway takes place is given in Movie 1 and Fig. 5. Movie 1 shows the evolution of $\alpha$, vertical and horizontal axes correspond to the $z$ and $r$ coordinates, respectively (see Fig 1); so, the top left corner corresponds to the top center point in the sample. Given that when thermal runaway occurs the decomposition rate increases considerably, frames are taken at constant degree of transformation intervals; otherwise, combustion would be like a flash in the movie.

In the supercritical region the system approaches the adiabatic temperature $T_{A D}$, i.e., the temperature the sample would reach under adiabatic conditions, ${ }^{31}$

$$
\Delta T_{A D} \equiv T_{A D}-T_{I s o}=\frac{q}{c}
$$

One can observe that, locally, the temperature approaches the adiabatic one $\left(T_{A D}=\right.$ $602^{\circ} \mathrm{C}$ ). Still, the maximum temperature is $30^{\circ} \mathrm{C}$ below $T_{A D}$. This difference is due to the low reaction enthalpy. Also, due to the local overheating, a temperature front is formed that propagates through the sample, this feature is clearer in Movie 1.

From Movie 1, we can observe that the combustion front is initiated at the top center and not at the bottom of the sample where it is in thermal contact with the furnace. The reason is that the vessel walls are a thermal sink that allows rapid heat dissipation thus preventing local overheating and, consequently, the formation of a combustion front there. For a sample mass near the critical value, the front is always initiated at the farthest location from the vessel walls, i.e., the top center of the sample, and propagates towards the vessel walls (for a closed vessel full of reactant the combustion front would initiate right in the center of the system).

\section{Critical condition for thermal explosion.}




\section{Dimensionless systems}

The model introduced in the previous section leads to a partial differential equation (PDE) coupled to a non-linear ordinary differential equation (ODE). As a result, thermal explosion is a complex system to treat both analytically and numerically. To simplify the analysis, simple geometries are assumed and the model is rewritten in a dimensionless form: ${ }^{31,32}$

$$
\begin{aligned}
& \frac{\partial \theta}{\partial \tau}=\left(\frac{\partial^{2} \theta}{\partial \xi^{2}}+\frac{N}{\xi} \frac{\partial \theta}{\partial \xi}\right)+\theta_{T} \frac{\partial \alpha}{\partial \tau} \\
& \frac{\partial \alpha}{\partial \tau}=\frac{1}{\theta_{T}} e^{\frac{\theta}{1+\varepsilon \theta}}(1-\alpha)
\end{aligned},
$$

where $N=0,1$ and 2 corresponds to a thin-film, an infinite cylinder and a sphere, respectively. The boundary conditions are:

$\theta(\sqrt{\delta})=\left.0 \quad \frac{\partial \theta}{\partial \xi}\right|_{\xi=0}=0 \Leftrightarrow \theta(0)=\theta_{\operatorname{Max}}$

The dimensionless temperature $\theta$, time $\tau$ and space coordinate $\xi$ are defined as:

$\theta \equiv \frac{E_{A}}{R_{G} T_{f}^{2}}\left(T-T_{f}\right), \tau \equiv \frac{t}{t_{i}}$ and $\xi \equiv \frac{r}{d_{i}}$,

where, $t_{i}$ is the thermal runaway induction time scale, i.e., a time scale for the time elapsed before the thermal runaway, ${ }^{39}$

$t_{i}=\left(\frac{q}{c} \frac{E_{A}}{R_{G} T_{f}^{2}} A e^{-\frac{E_{A}}{R_{G} T_{f}}}\right)^{-1}$

and $d_{i}$ is a scale of the width of the zone where the reaction rate is significant: $:^{31,32}$

$$
d_{i} \equiv \sqrt{\frac{\lambda}{\rho c} t_{i}} .
$$


The 1D system is fully described by three dimensionless parameters; namely: the Arrhenius parameter $\varepsilon$, the Todes or the Zeldovich parameter $\theta_{T}$ and the FrankKamenetskii parameter $\delta$ :

$\varepsilon \equiv \frac{R_{G} T_{f}}{E_{A}}$,

$\theta_{T} \equiv \frac{E_{A}}{R_{G} T_{f}^{2}} \frac{q}{c}=\frac{E_{A}}{R_{G} T_{f}^{2}}\left(T_{A D}-T_{f}\right)=\theta_{A D}$

$\delta \equiv \rho \frac{q}{\lambda} \frac{E_{A}}{R_{G} T_{f}^{2}} d^{2} A e^{-\frac{E_{A}}{R_{G} T_{f}}}=\left(\frac{d}{d_{i}}\right)^{2}$

where $d$ is a characteristic length. For a spherical vessel or an infinite cylinder it is the radius $(d=R)$ and for a thin-film is the film thickness $(d=H)$. Note that Todes parameter is the dimensionless adiabatic temperature.

Critical condition in the limit of no reactant consumption $\left(\theta_{T} \longrightarrow \infty\right)$

Historically, two different approaches have been followed to simplify the model: the stationary and the non-stationary approximations. ${ }^{31,32}$ In the stationary approach, the condition under which a stationary state is impossible establishes the critical condition. For a given symmetry the boundary problem can be solved to obtain the critical value. ${ }^{31,62-64}$ In particular, if we neglect the reactant consumption and we assume an infinite activation energy, the critical condition depends only on the FrankKamenetskii parameter (see appendix A):

$\delta_{c r}=C^{(1 D)}$

where subscript $c r$ stands for the parameter value at the threshold of ignition, and $C^{(I D)}$ is a constant that depends on the system's geometry: $C_{S p h e}^{(1 D)}=3.32$ for a spherical vessel, 
$C_{C y l}^{(1 D)}=2.0$ for an infinite cylinder and $C_{T F}^{(1 D)}=0.878$ for a thin film. The superscript (1D) stands for one-dimensional heat propagation. Above this critical value, $\delta>C$, ignition occurs.

The non-stationary approximation consist in describing the evolution of a spaceaveraged temperature $\bar{\theta}$ and transformation degree $\bar{\alpha}$. It has been shown that this approach allows to reduce PDE systems that exhibit a low-dimensional dynamics to an equivalent ODE system. ${ }^{65}$ Under this approximation, the dimensionless system, Eqs. (9) and (10), becomes: $:^{2,31,40,66}$

$$
\begin{aligned}
& \frac{\partial \bar{\theta}}{\partial \tau}=\theta_{T} \frac{\partial \bar{\alpha}}{\partial \tau}-\frac{B \bar{\theta}}{\delta} \\
& \frac{\partial \bar{\alpha}}{\partial \tau}=\frac{1}{\theta_{T}} e^{\frac{\bar{\theta}}{1+\varepsilon \bar{\theta}}}(1-\bar{\alpha})
\end{aligned}
$$

where $B$ is a constant that depends on the geometry of the system and the initial values of $\bar{\theta}$ and $\bar{\alpha}$ are zero. Parameter $B$ can be deduced by comparing the critical conditions delivered by the stationary and the non-stationary approximations in the limit case of infinite activation energy and no reactant consumption; $B=e \cdot C^{(1 D)}$ (see appendix B).

Eq. (16) has no analytical solution and its dynamics is controlled by two time scales; a time scale related to heat generation, $t_{i}$, and a time scale related to heat dissipation, $t_{D}$ (see appendix B):

$t_{D}^{(1 D)}=\frac{\rho c}{\lambda} \frac{d^{2}}{e \cdot C^{(1 D)}}$

So the critical condition can be expressed in terms of the ratio $t_{i} / t_{D}$ :

$$
t_{i, c r} / t_{D, c r}^{(1 D)}=e,
$$

Eqs. (15) and (18) are formally identical, i.e., both stationary and non-stationary approaches lead to the same critical condition for combustion. For $t_{i}>>t_{D}$ heat is 
efficiently removed and no local overheating occurs; this regime is the steady-state or subcritical solution. Conversely, when $t_{D}>t_{i}$ thermal explosion occurs and the local overheating results in a temperature rise that approaches the adiabatic temperature, this regime corresponds to the supercritical solution.

\section{Criterion to state the occurrence of a thermal runaway}

For $\theta_{\mathrm{T}}=\infty$, the transition between the subcritical and supercritical states can be established rigorously (see Appendix C); in the subcritical regime the system evolves to a stable steady state, while in the supercritical regime, the temperature is unbounded and it increases steadily with time. Conversely, for finite values of $\theta_{\mathrm{T}}$ due to reactant consumption the system always converges to a stable steady state. Thus, when reactant consumption is taken into account, Semenov's criteria based on the boundedness of the supercritical state can no longer be used..$^{36,43,67}$

For highly exothermic reactions and high activation energies $\left(\theta_{\mathrm{T}} \rightarrow \infty\right.$ and $\varepsilon \rightarrow$ 0) the separation between subcritical and supercritical regimes is sharp. The ignition temperature is well below the temperature of the supercritical state. In the subcritical state, the reaction heat is balanced by heat dissipation while in the supercritical state the reaction behaves nearly adiabatically: the heat evolved is some orders of magnitude larger than the heat dissipated. The time scale is also very different. It is some orders of magnitude smaller in the supercritical regime than in the subcritical regime. So, the particular criterion used to determine whether runaway occurs or not has little relevance. 
However, things are different when the heat of reaction or the activation energy is relatively low (typically below $10^{6} \mathrm{~J} / \mathrm{kg}$ ). Frank-Kamenetskii ${ }^{31}$ showed that the difference between the ignition and furnace temperatures is approximately,

$$
\Delta T_{i} \equiv T_{i}-T_{f} \approx \frac{R T_{f}^{2}}{E_{A}} .
$$

Thus, for low activation energies, $\Delta T_{i}$ will be high. On the other hand, for small values of the reaction enthalpy, the adiabatic temperature rise, $\Delta T_{A D}$, will also be small. Thus, in both cases the difference between the ignition and adiabatic temperatures will be relatively small and, therefore, the transition between the subcritical and supercritical regimes would be smooth. So, the critical condition for a thermal runaway depends on the particular criterion used to establish the boundary between both regimes. ${ }^{42,43}$

Thomas ${ }^{34}$ derived an approximate solution of Eq. (9) that takes into account reactant consumption. This solution diverges in a certain parameter region, so Semenov's criterion can be applied. Some authors ${ }^{36,42,68}$ studied the solution sensitivity at the limit of large activation energy by means of asymptotic analyses.

Some criteria are based on a geometrical property in the temperature profile. ${ }^{67,69}$ For instance, Thomas and Bowes ${ }^{70}$ and Adler and Enig ${ }^{2}$ proposed a criteria based on the existence of an inflection point before the local maximum, in the temperature-time and temperature-conversion profiles, respectively. This inflection point is related to a step temperature rise. Since initially the second derivative of the temperature is negative, a thermal runaway occurs provided that the second derivative is positive while the first derivative of temperature with respect to time is positive.

Several criteria are based on the parameter sensitivity, i.e., the solution becomes very sensitive to the values of the dimensionless control parameters ${ }^{43,67,69,71}$. For instance Bilous and Amundson ${ }^{72}$ considered the sensitivity of the maximum 
temperature, $\theta_{\mathrm{M}}$, with respect to Frank-Kamenetskii's parameter: the critical condition is given by the maximum of $d \theta_{\mathrm{M}} / d \delta$ (small deviations of $\delta$ from $\delta_{c r}$ result in the larger variations of $\theta_{\mathrm{M}}$ ). Zaldivar et $\mathrm{al}^{3}$ developed a criterion based on the system divergence: a runaway occurs when the divergence of the system (trace of the Jacobian matrix) is positive. A more complete overview of criteria is given by Varma et al. ${ }^{71}$ and Casson et al. $^{67,69}$

We propose a new criterion based on the evolution of $\phi$ with the conversion degree. In particular, we consider that criticality is defined by the existence of a local maximum in the $\phi$ versus $\bar{\alpha}$ curve in which $\phi>1$, i.e., during a time lapse heat generated by the reaction overcomes heat removal. This criterion is based on physical grounds and has the advantage of being intrinsic, i.e., it is not based on an arbitrary definition of the critical value of a given magnitude. Besides, it can be applied to the PDE model, Eqs. (1)-(3) as well as the approximate ODE system, Eq. (16). Precisely, $\phi$ for the ODE system can be derived by applying the normalization described in Eq. (11) into Eqs. (5) and (6):

$\phi=\delta \frac{e^{\frac{\bar{\theta}}{1+\varepsilon \bar{\theta}}}(1-\bar{\alpha})}{B \bar{\theta}}$.

Note, that $\phi$ is the ratio between the two terms that determine the evolution of the spaceaveraged temperature in Eq. (16). This is as expected, because these two terms are directly related to the contribution of heat generation and heat dissipation.

Our criterion is independent of the particular choice of the scaling used to derive the dimensionless system. Moreover, our criterion is equivalent to the often used criterion of Adler and Enig (see Appendix D).

Now, we will analyze how the particular choice of the runaway criterion affects the critical condition. First, we will determine the parameter range to be explored to 
account for most real cases. Most bonding energies are between 50 and $500 \mathrm{~kJ} / \mathrm{mol}$ (bond energies bellow $50 \mathrm{~kJ} / \mathrm{mol}$ are characteristic of weak interactions such as Van der Waals bonding). Assuming $T_{f}=600 \mathrm{~K}$, a variation of $E_{A}$ between 50 and $500 \mathrm{~kJ} / \mathrm{mol}$ results in a variation of $\varepsilon$ between 0.01 and 0.1 . Indeed there is no thermal runaway for $\varepsilon$ values above $1 / 4$ (Appendix C). ${ }^{35}$ As for the Todes parameter, Morbidelli and Varma ${ }^{73}$ showed that for first order reactions, no thermal runaway occurs for $\theta_{\mathrm{T}}$ values below 10 when $\varepsilon>0.15$ (this limit goes down to $\theta_{\mathrm{T}}=4$ for $\varepsilon=0$ ). So we will limit our numerical analysis to $\varepsilon$ values between 0 and 0.1 and $\theta_{\mathrm{T}}>10$.

In Fig. 6 we compare the critical value of Frank-Kamenetskii parameter determined using Thomas and Bowes, Bilous and Amundson, Zaldivar et al. and energy balance criteria. We consider three different values of $\varepsilon: 0,0.05$ and 0.1 . For given values of $\varepsilon$ and $\theta_{\mathrm{T}}$ Eq. (15) is solved numerically to determine the value of $\delta_{c r}$ that fulfils the corresponding criteria. We have assumed thin film geometry. As expected, in the limit $\theta_{\mathrm{T}} \rightarrow \infty$ all values converge to the values obtained using Semenov's criteria (Appendix C).

Lacey $^{42}$ stated that Thomas and Bowes, Adler and Enig, and Bilous and Amundson criteria deliver very similar results for moderate values of $\theta_{\mathrm{T}}$ and $\varepsilon$. Morbidelli et al. $^{43}$ confirmed this result and extended its validity to parametric sensitivity based on any system control parameter. This conclusion is also apparent in Fig. 6, where discrepancies are only significant for $\theta_{\mathrm{T}}<25$ or $\varepsilon \rightarrow 0.1$. The criterion of Zaldivar et al. is the one that exhibits the larger deviations. This criterion can be considered as a lower bound for the critical condition. The agreement between parametric sensitive (Bilous and Amundson) and the energy balance criteria is noteworthy - they virtually predict the same value of $\delta_{c r}$ in the entire parameter region except for values of $\varepsilon$ approaching 0.1 . 


\section{Accuracy of the non-stationary model}

The non-stationary model is the starting point for most analytical approaches. So to check its accuracy we will compare it to the exact PDE system, Eqs. (1)-(3). In the following, to determine $\delta_{c r}$ we will use the energy balance criteria because it can be applied to both systems. The results are plotted in Figs. 7, 8 and 9. In Fig. 7 we have plotted $\delta_{c r}$ as a function of $\theta_{\mathrm{T}}$ for $\varepsilon=0$ while in Fig. 8 we have plotted $\delta_{c r}$ as a function of $\theta_{\mathrm{T}}$ for $\theta_{\mathrm{T}}=100$. In Fig 9 we have plotted the percent deviations of the non-stationary model relative to the PDE system for $\theta_{\mathrm{T}}$ values between 10 and $10^{5}$ and for $\varepsilon$ values between 0 and 0.1 . From Figs. 7, 8 and 9 it is apparent that the non-stationary model is in a very nice agreement with the exact solution along the whole range of $\varepsilon$ and $\theta_{\mathrm{T}}$ values tested.

A noteworthy property of the ODE system, see Eq. (16), is that geometry only affects the numerical value of parameter $\delta_{c r}$ by a constant factor $1 / B$. Therefore, the functional dependence on $\varepsilon$ and $\theta_{T}$ is independent of the particular geometry. To verify this property we will consider two (1D) geometries; a thin film and an infinite cylinder. From Eq. (15) it can be stated that, in the limit $\theta_{\mathrm{T}}=\infty$ and $\varepsilon=0$, $\delta_{C r, T F} / C_{T F}^{(1 D)}=\delta_{C r, C y l} / C_{C y l}^{(1 D)}$. Thus, if this property holds, then $\delta_{C r, T F}=\delta_{C r, C y l} C_{T F}^{(1 D)} / C_{C y l}^{(1 D)}=0.439 \delta_{C r, C y l}$ for any value of $\varepsilon$ and $\theta_{T}$. In Figs. 7 and 8 we have plotted the value of $\delta_{c r}$ determined from the PDE system for a thin film and an infinite cylinder. Again, the agreement is remarkable. In Fig. 9 we have plotted the percent deviations of $0.439 \delta_{C r, C y l}$ with respect to $\delta_{C r, T F}$. Fig. 9 confirms this agreement for the whole range of of $\varepsilon$ and $\theta_{\mathrm{T}}$ values studied. Thus, the contribution of the geometry 
can be separated from the rest of parameters. We will take advantage of this property to derive the runaway critical condition for a finite cylinder.

\section{Accuracy of the existing critical conditions}

Several authors have derived analytical critical conditions using approximate solutions of Eq. (16). For instance Thomas ${ }^{34}$ derived a criterion that accounts for the reactant consumption (finite values of $\theta_{\mathrm{T}}$ ),

$$
\left.\delta\left\{1-2.85\left(\frac{n}{\theta_{T}}\right)^{2 / 3}\right\}\right|_{c r}=C^{(1 D)}
$$

where $n$ is the reaction order. A formally identical solution was obtained by Kassoy et al. $^{35}$ but replacing 2.85 by 2.946 . Using a different approach, Frank-Kamenetskii ${ }^{74}$ obtained,

$$
\left.\frac{\delta}{1+2.703\left(n / \theta_{T}\right)^{2 / 3}}\right|_{c r}=C^{(1 D)}
$$

Notice than in the limit of large values of $\theta_{T}$ Eqs. (21) and (22) are formally identical; they only differ in the value of the coefficient. Gray and Lee ${ }^{75}$ also obtained the same relationship but with a coefficient 2.946 instead of 2.703 .

Lacey $^{42}$ derived a critical condition that also takes into account finite values of the activation energy,

$$
\left.\delta\left\{1-2.946\left(\frac{n}{\theta_{T}}\right)^{2 / 3}-\frac{4}{9} \frac{n}{\theta_{T}} \ln \varepsilon\right\}\right|_{c r}=C^{(1 D)}
$$

Finally Babushok et al. ${ }^{36}$ developed the more accurate series expansion for small values of $1 / \theta_{T}$ and $\varepsilon$, 
$\left.\delta(1-\varepsilon)\left\{1-2.946(1+2 \varepsilon)\left(\frac{n}{\theta_{T}}\right)^{2 / 3}+\frac{4}{9}(1+6 \varepsilon) \frac{n}{\theta_{T}} \ln \theta_{T}\right\}\right|_{c r}=C^{(1 D)}$

In Fig. 7 we have plotted the prediction of $\delta_{\text {cr }}$ calculated from all these condition but Lacey's condition (it diverges in the limit $\varepsilon \rightarrow 0$ ). From Figs 7, 8 and 9 it is apparent that all the models provide an accurate prediction for highly exothermic reactions and high activation energies (small values of $1 / \theta_{T}$ and $\varepsilon$ ), but inaccuracies are significant for less exothermic reactions or low activation energies. As expected, the model of Babushok et al. is the one that better approaches the exact solution. Only, Lacey and Babushok et al. critical conditions take into account finite values of the activation energy. Since the remaining models have been derived assuming infinite activation energy they are quite inaccurate when low activation energies are considered. Also, despite the fact that finite activation energies have been taken into account, the model of Lacey fails to provide an accurate prediction for low activation energies.

Two aspects affect the observed differences: the accuracy of the approximations assumed in the derivation of the model and the criterion taken to establish the occurrence of a thermal runaway. Note that despite the fact that all thermal runaway criteria converge to the same value for $\theta_{\mathrm{T}}=\infty$ (Fig. 6), deviations of the different models are quite significant for $\theta_{\mathrm{T}}=\infty$ (see Fig. 9). Hence, the main source of the deviations is related to the accuracy of the models. For this reason, the finest development is the one that gives the most accurate prediction.

\section{Thermal runaway condition for 1D geometry: analysis of combustion in thin-films}

Despite the fact that Babushok et al. critical condition is the most accurate model, deviations are still relevant for low reaction enthalpies or low activation 
energies. Also, the previous critical conditions exhibit a similar dependence on $\theta_{\mathrm{T}}$ that qualitatively reproduces the observed dependence on $\theta_{\mathrm{T}}$. As for the dependence on parameter $\varepsilon$, it can be shown, (see Appendix C) that in the limit of $\theta_{\mathrm{T}} \rightarrow \infty$ the critical condition is approximately $\left.\delta(1-\varepsilon)\right|_{c r}=C^{(1 D)}$, which agrees perfectly with Babushok et al., Eq. (24). So, combining the latter result with the functional dependence of the existing criteria we obtain:

$$
\left.\delta(1-\varepsilon)\left(1-2.25(1+3.76 \varepsilon)\left(\frac{n}{\theta_{T}}\right)^{2 / 3}\right)\right|_{c r}=C,
$$

parameters 2.25 and 3.76 have been fitted using a numerical method that minimizes the deviations relative to the exact solution in the $0 \leq \varepsilon \leq 0.1$ and $\theta_{T} \geq 10$ range.

In Figs. 7 and 8 one sees that Eq. (25) is the one that best fits the exact solution. To check the accuracy of our approximation, we have calculated the percent error in the calculation of $\delta_{c r}$. The result is plotted in Fig 10 where it is apparent that our critical condition delivers an accurate prediction within the whole range of parameters; the relative error in the calculation of the critical thickness is less than $5 \%$ and below $3 \%$ for $\varepsilon<0.05$. An inaccuracy below $3 \%$ is negligible, especially when the approximate nature of the model itself and the uncertainties of the system parameters are taken into account.

It is well-known that a critical size exists below which no thermal runaway occurs. $^{1,61}$ Now, we are in the position to evaluate the critical thickness for thermal explosion to occur in films when they are heated homogenously. In particular, we will analyze the possibility to synthetize metal oxide thin-films via volume combustion synthesis. From Figs. 6 and 7 it is apparent that the value of $\delta_{c r}$ is between 1 and 2 . Once we now $\delta_{c r}$, we can determine the critical film thickness from Eq. (14): 


$$
H_{c r}=\sqrt{\frac{\delta_{c r}}{\theta_{T}} a t_{R}},
$$

where $a$ is the thermal diffusivity $(a \equiv \lambda / \rho c)$ and $t_{R}$ is a reaction time scale,

$$
t_{R}=\left(A e^{-E_{A} / R_{G} T_{f}}\right)^{-1}
$$

Now, we are going to estimate the physically reasonable lower bound of the critical thickness. For solids $\rho c \approx 3 \times 10^{6} \mathrm{~J} / \mathrm{m}^{3} \mathrm{~K}$ and the lowest conductivities of solid materials are around $\lambda=0.1 \mathrm{~W} /(\mathrm{m} \cdot \mathrm{K}),{ }^{76}$ so, the minimum value of $a$ is around $3 \times 10^{-8} \mathrm{~m}^{2} / \mathrm{s}$. For combustion synthesis to be of practical interest reaction time should be significantly longer than combustion time; considering reactions that will last less than one hour in the absence of any overheating is of no use, so we take $3600 \mathrm{~s}$ as lower bound for $t_{R}$. Adiabatic temperature rise in metal oxide precursors is of the order of few hundred Kelvin; in the most optimistic case, around $1000 \mathrm{~K}$. Typically $T_{f} \approx 600 \mathrm{~K},{ }^{25,26}$ so we take $300 \mathrm{~K}$ as a lower bound. Activation energies are of the order of $100 \mathrm{~kJ} / \mathrm{mol}$, we take as upper estimation $E_{A}=500 \mathrm{~kJ} / \mathrm{mol}$. Then $\theta_{\mathrm{T}}$ is around 670 in the most favorable conditions for solid-state reactions. Therefore, in the best case scenario the critical film thickness is around $400 \mu \mathrm{m}$. Thus volume combustion synthesis of oxide thin-films is at least very difficult not to say impossible. This conclusion is in agreement with experiments showing precursors that experience thermal explosion in the form of powders, but not in the form of films. ${ }^{26,53,77-79}$

\section{Combustion criterion for a finite size cylindrical vessel.}


As we have seen in section 4.4, the contribution of geometry can be separated from that of the rest of parameters. Thus, Eq. (25) is still valid for a 2D geometry but we need to determine the geometrical factor for a finite cylinder, $C^{(2 D)}$.

The contribution of the geometry is related to heat transport through diffusion. Diffusion can be approximately addressed by considering that two paths compete in parallel; an axial path and a radial path. Thus the diffusion length is,

$$
\frac{1}{d^{(2 D)}}=\frac{1}{H}+\frac{1}{R}
$$

and the diffusion time for a finite cylinder is given by,

$$
\frac{1}{t_{D}^{(2 D)}}=\frac{1}{t_{D, T F}^{(1 D)}}+\frac{1}{t_{D, C y l}^{(1 D)}}
$$

Combining Eqs. (17) and (29) and keeping in mind that $C_{T F}^{(1 D)}=0.878$ and $C_{C y l}^{(1 D)}=2.0$, we obtain,

$$
\frac{C^{(2 D)}}{\left(d^{(2 D)}\right)^{2}}=\frac{0.878}{H^{2}}+\frac{2}{R^{2}} .
$$

$C^{(2 D)}$ is obtained by substituting Eq. (28) into (30),

$$
C^{(2 D)}=\frac{0.878 R^{2}+2 H^{2}}{(H+R)^{2}} .
$$

To check the accuracy of Eq. (31), in Fig. 11 we have plotted the value of $C^{(2 D)}$ delivered from Eq. (31) and we compare it to the value determined from the exact model Eqs. (1)-(3). Specifically, for different values of the aspect ratio and of the parameters $\varepsilon$ and $\theta_{\mathrm{T}}$, we determine numerically the value of $\delta_{c r}$ at the threshold of combustion. Then, $C^{(2 D)}$ is obtained from Eq. (25). Values of $\varepsilon$ vary from 0.02 to 0.03 and the values of $\theta_{\mathrm{T}}$ are between 10 and $2.8 \times 10^{4}$. From Fig. 11 it is clear that Eq. (31) gives an accurate estimation of the geometrical factor for a finite cylinder. Note, that as expected, this parameter depends only on the cylinder aspect ratio, $H / R$. Also, in Fig. 11 
we have plotted the usual approximation of assuming a sphere of the same volume, so that the $2 \mathrm{D}$ model is reduced to a $1 \mathrm{D}$ model ${ }^{6}$ This approach is equivalent to assume a constant $C^{(2 D)}=3.32$. From Fig. 11 one can verify that this approach is only useful for $H / R$ values near 1 . A more accurate approach is based on the Kondratiev theory of regular mode. ${ }^{31,80}$ Under this approach, heat transfer in the cylindrical vessel can be approximate to a sphere of radius $3.07880 / \sqrt{(1.54 / H)^{2}+(2.3572 / R)^{2}}$. From Fig. 11 one can verify that the value of $C^{(2 D)}$ derived using this approach is nearly as accurate as Eq. (31). Indeed, it can be shown that both approaches lead to the same formal dependence for $C^{(2 D)}$, Eq. (31), but with slightly different values of the coefficients: 0.831 and 1.95 instead of 0.878 and 2.

The steps to determine if a thermal runaway will occur are summarized in Figure 12.

Finally, from Eqs. (25), (28) and (31) one can determine the sample critical mass:

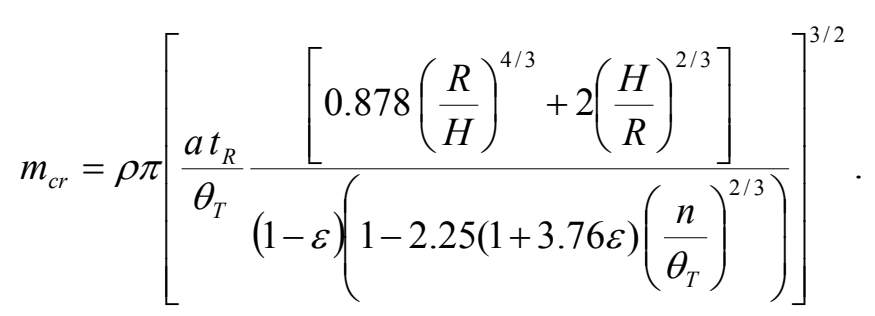

Eq. (32) has been determined assuming that the vessel containing the sample is not covered by a lid. The case of a sample that fills a closed vessel is equivalent but with $H$ being half the sample height.

We have applied Eq. (32) to determine the critical mass for a cylindrical crucible filled with $\mathrm{Y}(\mathrm{TFA})_{3}$ powders. The physical parameters of $\mathrm{Y}(\mathrm{TFA})_{3}$ are indicated in Table 1. For a furnace temperature of $290^{\circ} \mathrm{C}$ and a cylinder of radius $2.5 \mathrm{~mm}$ we obtain a critical mass of $41.0 \mathrm{mg}$ that agrees very well with the value of $41.2 \mathrm{mg}$ obtained from 
the numerical simulations and in a fair agreement with the critical value around $36 \mathrm{mg}$ determined from experiments.

From Eq. (28) one can realize that, when one of the crucible dimensions goes to zero, $d^{(2 D)}$ approaches the vanishing dimension; i.e., the shortest dimension becomes a very effective path for heat dissipation. Thus, thermal explosion is inhibited when the radius or the height are too small. This is the case of thin-films analyzed in the previous section. In thin-films heat is dissipated very efficiently towards the substrate due to the high surface to thickness aspect ratio.

Similarly, in cylindrical vessels, the smaller dimension will limit the occurrence of combustion. In our case, the $\mathrm{Al}_{2} \mathrm{O}_{3}$ crucibles have an internal radius of $2.5 \mathrm{~mm}$ and a height of $4.5 \mathrm{~mm}$. So, when completely filled, the limiting dimension is the radial one. From Eq. (25) one can determine the critical temperature for an infinite cylinder. Using the parameters of $\mathrm{Y}(\mathrm{TFA})_{3}$ and a radius of $2.5 \mathrm{~mm}$ we obtain a critical temperature of $281.4^{\circ} \mathrm{C}$, i.e., no matter how full of $\mathrm{Y}(\mathrm{TFA})_{3}$ the crucible is, combustion will be impossible in cylindrical crucibles of radius $2.5 \mathrm{~mm}$ at temperatures below $281.4^{\circ} \mathrm{C}$. If we take into account the finite size of the crucible, Eq. (28), the critical temperature is $283.5^{\circ} \mathrm{C}$. Again this result is in good agreement with the numerical and experimental results presented in section 3. In particular, we have observed numerically and experimentally the occurrence of combustion for a temperature of $290^{\circ} \mathrm{C}$, but never for a temperature of $280^{\circ} \mathrm{C}$.

\section{Conclusions}

- We have introduced a new criterion to establish the boundary of thermal runaway. This criterion is based on the evolution of the ratio between heat 
generation and heat removal rates. Under the non-stationary approximation this criterion coincides with the well-known criterion of Adler and Enig.

- The main advantages of this new criterion is that it is based on physical grounds, not on an arbitrary choice of the magnitude values or the relevant parameters, it is independent of the particular scales used to define the dimensionless variables and it can be applied to the exact PDE system as well as to the approximate nonstationary ODE model.

- The non-stationary model gives a very accurate prediction of the critical condition for thermal explosion. The discrepancy between the models and the numerical solution is not due to the choice of the particular criteria to establish the boundary of thermal explosion but to the particular approximation used in the model development.

- From a fit to the exact solution, we have developed a new critical condition for the combustion onset. This critical condition is accurate for any realistic combustion event; event for low enthalpy or for low energy activation reactions, where other criteria fail.

- We have extended the applicability of this critical condition to a cylindrical vessel without any restriction on its aspect ratio. This approach leads to a prediction of the minimum sample mass or sample size.

- The sample aspect ratio plays a crucial role since thermal explosion is harder to achieve when one of the dimensions vanishes. In particular, it is concluded that thermal explosion of submicrometric films is virtually impossible.

- Numerical simulations were compared to experiments carried out on metalorganic powders that decompose inside a crucible. Although numerical 
simulations fail to describe accurately the complete reaction course, they are able to predict the temperature and mass thresholds for thermal explosion.

\section{Appendix A. Stationary approach.}

As we have seen, in the subcritical parameter region, a stable low temperature state exists where heat generation is compensated by heat dissipation: so, the time derivative of the temperature in Eq. (9) can be neglected. Furthermore, Frank-Kamenetskii assumed that $\varepsilon \theta<<1$ and $\theta_{T}>>1$. These are reasonable approximations for high enthalpy system where $T_{A D} \gg T_{f}$. Besides, Todes parameter is the quotient between the reaction and ignition time scales, ${ }^{61}$ therefore a large value of $\theta_{T}$ is consistent with the completion of the reaction in a much shorter time when combustion takes place. Under these assumptions the system is described by, ${ }^{31,63}$

$\left(\frac{\partial^{2} \theta}{\partial \xi^{2}}+\frac{N}{\xi} \frac{\partial \theta}{\partial \xi}\right)+e^{\theta}=0$

$\left.\frac{\partial \theta}{\partial \xi}\right|_{\xi=0}=0, \quad \theta(\sqrt{\delta})=0$

For $N=0$ (thin film) Eq. (A.1) has a solution, ${ }^{63}$

$\theta=2 \ln \left[k \operatorname{sech}\left(\frac{k}{\sqrt{2}} \xi\right)\right]$

where $k$ is an integration constant. The value of $k$ can be determined from the boundary condition,

$\theta(\sqrt{\delta})=0$ or $\delta=2\left(\frac{1}{k} \operatorname{arcosh}[k]\right)^{2}$

Thermal explosion occurs when there is no solution for the stationary state. From Fig. A.1 it is apparent that above a certain value, $\delta_{c r}$, there is no solution for Eq. (A.3). 
Therefore, the critical condition is given by the maximum in Fig. A.1 that corresponds to $k=1.81017$. Finally, substituting this value of $k$ into Eq. (A.3) we obtain the critical value $\delta_{c r}=0.878458$

Similarly, for $N=1$ (infinite cylinder) the solution of Eq. (A.1) is, ${ }^{63}$

$\theta=2 \ln \left[\frac{4 k}{2 k^{2}+\xi^{2}}\right]$

Again, from the boundary condition we determine the value of $k$,

$\theta(\sqrt{\delta})=0$ or $2 k^{2}-4 k+\delta=0$.

Eq. (A.5) has a solution provided that $\delta \geq 2$. Therefore, for an infinite cylinder the critical condition becomes $\delta_{c r}=2$.

Finally, for $N=2$ (sphere) Eq. (A.1) has no analytical solution. Chambré ${ }^{62}$ has shown that this particular equation has been solved in astrophysical problems and that the critical condition for a sphere is $\delta_{c r}=3.32$.

\section{Appendix B. Non-stationary approach.}

Under the non-stationary approach and assuming $\varepsilon \theta<<1$ and $\theta_{T}>>1$, Eqs. (1) and (3) reduce to ${ }^{31,41}$

$\frac{\partial \bar{\theta}}{\partial t}=-\frac{\bar{\theta}}{t_{D}^{(1 D)}}+\frac{e^{\bar{\theta}}}{t_{i}}$

where $t_{D}^{(1 D)}$ is a time scale related to heat diffusion,

$t_{D}^{(1 D)}=\frac{\rho c}{\lambda} \frac{d^{2}}{B}$ 
Therefore, two time scales govern the dynamics of Eq. (B.1): a time scale related to the induction period, $t_{i}$, and a time scale related to heat dissipation, $t_{D}{ }^{2,71}$ Thus the critical condition for ignition is of the form

$t_{D, c r}^{(1 D)} / t_{i, c r}=$ constant

One sees that $t_{D, c r}^{(1 D)} / t_{i, c r}=\delta / B$, thus, Eqs. (15) and (B.3) are equivalent. If we replace the time in Eq. (B.1) by the dimensionless time, we obtain,

$\frac{\partial \bar{\theta}}{\partial \tau}=-\frac{B \bar{\theta}}{\delta}+e^{\bar{\theta}}$

And imposing a zero time derivative we obtain the steady-state solution,

$\frac{B}{\delta}=\frac{e^{\bar{\theta}}}{\bar{\theta}}$

So, there is a subcritical solution provided that Eq. (B.5) has a solution. In Fig. B.1 we have plotted the right hand side of Eq. (B.5) as a function of $\bar{\theta}$. One can verify that above a certain critical value of $\delta$, Eq. (B.5) has no solution. This critical value corresponds to the minimum of the function $e^{\bar{\theta}} / \bar{\theta}$ that is $\bar{\theta}_{c r}=1$, Therefore, the critical condition is

$\delta_{c r}=B / e$

By comparing Eq. (B.6) with the critical condition delivered by the stationary approach, Eq. (15), one can determine the value of $B$ for different geometries,

$B=e \cdot C^{(1 D)}$

and the diffusion time scale becomes,

$t_{D}^{(1 D)}=\frac{\rho c}{\lambda} \frac{d^{2}}{e \cdot C^{(1 D)}}$

So the critical condition for ignition can be expressed as, 
$t_{i, c r} / t_{D, c r}^{(1 D)}=e$

According to Eq. (1), the dependence on geometry is related to the heat dissipation term, so $t_{D}^{(1 D)}$ accounts for the contribution of the geometry. Conversely, all the parameters related to heat generation are included in $t_{i}$. So the diffusion and induction time scales account for the separated contribution of heat dissipation and heat generation, respectively.

Appendix C. Approximate critical condition in the limit $\theta_{\mathrm{T}} \rightarrow \infty$.

In the limit case $\theta_{\mathrm{T}} \rightarrow \infty$, Eq. (16) reduces to: ${ }^{31,40}$

$$
\frac{\partial \bar{\theta}}{\partial \tau}+\frac{B \bar{\theta}}{\delta}=e^{\bar{\theta}} / 1+\varepsilon \bar{\theta}
$$

Eq. (C.1) is equivalent to neglecting reactant consumption. Eq. (C.1) has two different asymptotic solutions: a stationary one that corresponds to the subcritical solution and a divergent one that is related to the supercritical regime. Therefore, the combustion criterion corresponds to the boundary between these two regimes. This rigorous criterion cannot be applied in the case of finite values of $\theta_{\mathrm{T}}$ because reactant consumption ensures that the system will always evolve to a stationary solution.

The stationary solution of Eq. (C.1) is given by

$$
\frac{\partial \bar{\theta}}{\partial \tau}=0 \Leftrightarrow \frac{B}{\delta}=f(\bar{\theta}) \equiv \frac{e^{\bar{\theta} / 1+\varepsilon \bar{\theta}}}{\bar{\theta}} .
$$

Likewise in Appendix B, the function $f(\bar{\theta})$ has a local minimum (see Fig. B.1). Thus for the $B / \delta$ values that lay below this minimum there is no stationary solution, i.e., the temporal evolution of $\bar{\theta}$ diverges. Thus, the critical condition corresponds to the local minimum of $f(\bar{\theta})$ : 
$\frac{d f(\bar{\theta})}{d \bar{\theta}}=0 \Leftrightarrow(1+\varepsilon \bar{\theta})^{2}=\bar{\theta}$

The solution of Eq. (C.3) is

$$
1+\varepsilon \bar{\theta}=\frac{1-\sqrt{1-4 \varepsilon}}{2 \varepsilon} .
$$

Note that Eq. (C.3) has no solution for $\varepsilon>1 / 4$, i.e., for $\varepsilon>1 / 4$ there is no supercritical solution. Thus, the critical condition becomes:

$\left.\delta \frac{e^{1+\varepsilon \bar{\theta}}}{(1+\varepsilon \bar{\theta})^{2}}\right|_{c r}=B=e \cdot C^{(1 D)}$

If we substitute Eq. (C.4) into Eq. (C.5) and we perform a Taylor series expansion for small values of $\varepsilon$ we obtain:

$\left.\delta e\left(1-\varepsilon-\frac{\varepsilon^{2}}{2}-\frac{5 \varepsilon^{3}}{6}+\mathrm{O}\left(\varepsilon^{4}\right)\right)\right|_{c r}=e \cdot C^{(1 D)}$.

And finally, in a first order approximation the critical condition is:

$\left.\delta(1-\varepsilon)\right|_{c r}=C^{(1 D)}$.

\section{Appendix D. Equivalence between energy balance and Adler and Enig criteria.}

According to Adler and Enig criterion, ${ }^{2,42}$ in the subcritical regime $d^{2} \bar{\theta} / d \bar{\alpha}^{2}<0$ a thermal runaway occurs when for a time lapse $d^{2} \bar{\theta} / d \bar{\alpha}^{2}>0$ while $d \bar{\theta} / d \tau>0$. Thus, the occurrence of a thermal runaway involves a local minimum of the function $d \bar{\theta} / d \bar{\alpha}$. Besides, from Eq. (16),

$\frac{d \bar{\theta}}{d \bar{\alpha}}=\theta_{T}\left(1-\frac{B \bar{\theta}}{\delta e^{\frac{\bar{\theta}}{1+\varepsilon \bar{\theta}}(1-\bar{\alpha})}}\right)$.

and combining Eqs. (20) and (D.1), 


$$
\frac{d \bar{\theta}}{d \bar{\alpha}}=\theta_{T}\left(1-\frac{1}{\phi}\right) .
$$

Thus, the criterion of Adler and Enig is equivalent to a local maximum of the function $\phi$ versus conversion. As for the condition $d \bar{\theta} / d \tau>0$, note that the heat balance criterion imposes that heat generation must overcome heat generation. Thus, from Eq. (20)

$$
\phi=\delta \frac{e^{\frac{\bar{\theta}}{1+\varepsilon \bar{\theta}}}(1-\bar{\alpha})}{B \bar{\theta}}>1 .
$$

So, combining Eqs. (16) and (D.3),

$$
\frac{\partial \bar{\theta}}{\partial \tau}=e^{\frac{\bar{\theta}}{1+\varepsilon \bar{\theta}}}(1-\bar{\alpha})-\frac{B \bar{\theta}}{\delta}>0
$$

Under the non-stationary approach both criteria are equivalent but with the advantage that the energy balance criterion does not depend on the particular choice of the dimensionless system and can be applied to the PDE model.

\section{Acknowledgements}

This work was partially funded by the Spanish Programa Nacional de Materiales through project MAT2014-51778-C2-2-R, by the Generalitat de Catalunya contract No. 2014SGR-00948 and by the Universitat de Girona contract No. MPCUdG2016/059.

\section{Notation}

A Pre-exponential constant, $\mathrm{s}^{-1}$

$a \quad$ thermal diffusivity, $a \equiv \lambda / \rho c, \mathrm{~m}^{2} / \mathrm{s}$.

c Specific heat capacity, $\mathrm{J} /(\mathrm{kg} \cdot \mathrm{K})$

$B \quad$ Geometrical factor of the non-stationary model, Eq. (16). 
C Geometrical factor of the critical condition, Eqs. (15) and (25).

$d \quad$ characteristic linear dimension of Frank-Kamenetskii parameter, Eq. (14), m

$d_{i} \quad$ a scale of the width of the reaction zone, Eq. (13), m

$E_{A} \quad$ Activation energy, $\mathrm{J} / \mathrm{mol}$

$H \quad$ Sample height or film thickness, $\mathrm{m}$

$m \quad$ Sample mass, $\mathrm{kg}$

$n \quad$ Reaction order

$N \quad$ Integer related to the geometry of the diffusion, Eq. (9).

$q \quad$ Specific heat of reaction (positive for exothermic reactions), $\mathrm{J} / \mathrm{kg}$

$I_{\text {Gen }} \quad$ Heat generation rate, $\mathrm{J} / \mathrm{s}$

$I_{\text {Dis }} \quad$ Heat dissipation rate, $\mathrm{J} / \mathrm{s}$

$R_{G} \quad$ Universal gas constant, $\mathrm{J} /(\mathrm{K} \cdot \mathrm{mol})$

$R \quad$ Inner radius of a cylindrical or spherical reaction vessel, $\mathrm{m}$

$r \quad$ Radial space coordinate, $\mathrm{m}$

$t \quad$ Time, $\mathrm{s}$

$t_{D} \quad$ Thermal diffusion time scale, Eq. (B.2), $\mathrm{s}$

$t_{i} \quad$ Time scale of the adiabatic induction period, Eq. (12), s

$t_{R} \quad$ Time scale of the reaction, Eq. (27), $\mathrm{s}$

$T \quad$ Temperature, $\mathrm{K}$

$T_{A D} \quad$ Adiabatic temperature, Eq. (8), $\mathrm{K}$

$\triangle T_{A D} \quad$ Adiabatic temperature rise, Eq. (8), $\mathrm{K}$

$T_{f} \quad$ Temperature of the vessel containing the system, $\mathrm{K}$

$T_{i} \quad$ Approximate ignition temperature, $\mathrm{K}$

$V \quad$ Volume of the sample, $\mathrm{m}^{3}$.

$S \quad$ Surface that encloses the sample, $\mathrm{m}^{2}$ 
$z \quad$ Vertical space coordinate, $\mathrm{m}$

$\lambda \quad$ Thermal conductivity, $\mathrm{W} /(\mathrm{m} \cdot \mathrm{K})$

$\rho \quad$ Density, $\mathrm{kg} / \mathrm{m}^{3}$

\section{Dimensionless parameters}

$\alpha \quad$ Degree of transformation or conversion fraction

$\bar{\alpha} \quad$ Volume averaged degree of transformation

$\varepsilon \quad$ Arrhenius parameter, Eq. (14)

$\delta \quad$ Frank-Kamenetskii parameter, Eq. (14)

$\xi \quad$ Space coordinate, Eq. (11)

$\theta \quad$ Temperature, Eq. (11)

$\theta_{T} \quad$ Todes parameter, Eq. (14)

$\phi \quad$ Ratio between the heat generation and the heat dissipation rates, Eq. (7).

$\tau \quad$ Time, Eq. (11)

\section{Subscripts and superscripts}

cr critical, parameter value at the runaway threshold

(1D) 1D model

(2D) 2D model

TF 1D vessel geometry in the limit case $R>>H$ (thin film or semi-infinite slab)

Cyl 1D vessel geometry in the limit case $H \gg>R$ (infinite cylinder)

Sph Spherical vessel 


\section{Literature Cited}

1. Merzhanov AG, Khaikin BI. Theory of combustion waves in homogeneous media. Prog Energy Combust Sci. 1988;14(1):1-98.

2. Adler J, Enig JW. The critical conditions in thermal explosion theory with reactant consumption. Combust Flame. 1964;8(2):97-103.

3. Zaldívar JM, Cano J, Alós MA, et al. A general criterion to define runaway limits in chemical reactors. J Loss Prev Process Ind. 2003;16(3):187-200.

4. Roduit B, Xia L, Folly P, et al. The simulation of the thermal behavior of energetic materials based on DSC and HFC signals. $J$ Therm Anal Calorim. 2008;93(1):143-152.

5. Krause G. Volume-Dependent Self-Ignition Temperatures for Explosive Materials. Propellants, Explos Pyrotech. 2012;37(1):107-115.

6. Roduit B, Hartmann M, Folly P, Sarbach A, Brodard P, Baltensperger R. Determination of thermal hazard from DSC measurements. Investigation of selfaccelerating decomposition temperature (SADT) of AIBN. J Therm Anal Calorim. 2014;117(3):1017-1026.

7. Dellavedova M, Pasturenzi C, Gigante L, Lunghi A. Kinetic Evaluations for the Transportation of Dangerous Chemical Compounds. Chem Eng Trans. 2012;26:585-590.

8. Boddington T, Cottrell A, Laye PG. A numerical model of combustion in gasless pyrotechnic systems. Combust Flame. 1989;76(1):63-69.

9. Zinn J, Mader CL. Thermal Initiation of Explosives. $J$ Appl Phys. $1960 ; 31(2): 323$.

10. Rogers RN. Thermochemistry of explosives. Thermochim Acta. 1975;11(2):131139. 
11. Victor AC. Simple Calculation Methods for Munitions Cookoff times and temperatures. Propellants, Explos Pyrotech. 1995;20(5):252-259.

12. Wang Q, Ping $P$, Zhao X, Chu G, Sun J, Chen C. Thermal runaway caused fire and explosion of lithium ion battery. J Power Sources. 2012;208:210-224.

13. Balakrishnan PG, Ramesh R, Kumar TP. Safety mechanisms in lithium-ion batteries. J Power Sources. 2006;155(2):401-414.

14. Kotoyori T. Critical Temperatures for the Thermal Explosion of Chemicals (1st edition). Vol 7. 1st ed. Amsterdam: Elsevier; 2005.

15. Griffiths JF, Barnard JA. Flame and Combustion. 3rd ed. London: CRC Press; 1995.

16. Morsi K. The diversity of combustion synthesis processing: a review. J Mater Sci. 2011;47(1):68-92.

17. Moore JJ, Feng HJ. Combustion synthesis of advanced materials: Part I. Reaction parameters. Prog Mater Sci. 1995;39(4-5):243-273.

18. Varma A, Rogachev AS, Mukasyan AS, Hwang S. Combustion Synthesis of Advanced Materials: Principles and Applications. Adv Chem Eng. 1998;24:79226.

19. Patil KC, Aruna ST, Mimani T. Combustion synthesis: an update. Curr Opin Solid State Mater Sci. 2002;6(6):507-512.

20. Mossino P. Some aspects in self-propagating high-temperature synthesis. Ceram Int. 2004;30(3):311-332.

21. Yeh CL. Combustion Synthesis: Principles and Applications. In: Buschow KHJ, Cahn RW, Flemings MC, et al., eds. Encyclopedia of Materials: Science and Technology (2nd edition). Oxford: Elsevier; 2010:1-8.

22. Striker T, Ruud JA. Effect of Fuel Choice on the Aqueous Combustion Synthesis 
of Lanthanum Ferrite and Lanthanum Manganite. $J$ Am Ceram Soc. 2010;93(9):2622-2629.

23. Reeves RV, Rodriguez MA, Jones ED, Adams DP. Condensed-Phase and Oxidation Reaction Behavior of Ti/2B Foils in Varied Gaseous Environments. $J$ Phys Chem C. 2012;116(33):17904-17912.

24. Wang J, Besnoin E, Knio OM, Weihs TP. Effects of physical properties of components on reactive nanolayer joining. J Appl Phys. 2005;97(11):114307.

25. Kim MG, Kanatzidis MG, Facchetti A, Marks TJ. Low-temperature fabrication of high-performance metal oxide thin-film electronics via combustion processing. Nat Mater. 2011;10(5):382-388.

26. Sanchez-Rodriguez D, Farjas J, Roura P, et al. Thermal Analysis for Low Temperature Synthesis of Oxide Thin Films from Chemical Solutions. J Phys Chem C. 2013;117(39):20133-20138.

27. Rabinovich OS, Grinchuk PS, Andreev MA, Khina BB. Conditions for combustion synthesis in nanosized Ni/Al films on a substrate. Phys $B$ Condens Matter. 2007;392(1-2):272-280.

28. Sánchez-Rodríguez D, Eloussifi H, Farjas J, Roura P, Dammak M. Thermal gradients in thermal analysis experiments: Criterions to prevent inaccuracies when determining sample temperature and kinetic parameters. Thermochim Acta. 2014;589:37-46.

29. Phung PV, Hardt AP. Ignition characteristics of gasless reactions. Combust Flame. 1974;22(3):323-335.

30. Thiers L, Mukasyan AS, Varma A. Thermal explosion in Ni-Al system: influence of reaction medium microstructure. Combust Flame. 2002;131(1-2):198-209.

31. Frank-Kamenetskii DA. Diffusion and Heat Exchange in Chemical Kinetics (2nd 
edition). New Jersey: Princeton University Press; 1955.

32. Merzhanov AG, Averson AEE. The present state of the thermal ignition theory: An invited review. Combust Flame. 1971;16(1):89-124.

33. Victor AC. Equations for Predicting Cookoff Ignition Temperatures, heating times, and violence. Propellants, Explos Pyrotech. 1997;22(2):59-64.

34. Thomas PH. Effect of Reactant Consumption on the Induction Period and Critical Condition for a Thermal Explosion. Proc R Soc A Math Phys Eng Sci. 1961;262(1309):192-206.

35. Kassoy DR, Liñan A. The influence of reactant consumption on the critical conditions for homogeneous thermal explosions. $Q J$ Mech Appl Math. 1978;31(1):99-112.

36. Babushok VI, Goldshtein VM, Sobolev VA. Critical Conditions for Thermal Explosion with Reactant Consumption. Combust Sci Technol. 1990;70(1-3):8189.

37. Gonçalves de Azevedo F, Griffiths JF, Cardoso SSS. Effects of kinetic and transport phenomena on thermal explosion and oscillatory behaviour in a spherical reactor with mixed convection. Phys Chem Chem Phys. 2014;16(42):23365-23378.

38. Gray P, Harper MJ. Thermal explosions. Part 1.-Induction periods and temperature changes before spontaneous ignition. Trans Faraday Soc. 1959;55:581-590.

39. Todes OM, Melentjew PV. The theory of heat explosion II Heat explosion for mono-molecular reactions. Acta Physicochim URSS. 1939;11(2):153-180.

40. Squire W. A mathematical analysis of self-ignition. Combust Flame. 1963;7:1-8.

41. Gray P, Lee PR. Thermal explosions and the effect of reactant consumption on 
critical conditions. Combust Flame. 1965;9(2):201-203.

42. Lacey AA. Critical behaviour of homogeneous reacting systems with large activation energy. Int J Eng Sci. 1983;21(5):501-515.

43. Morbidelli M, Varma A. A generalized criterion for parametric sensitivity: Application to thermal explosion theory. Chem Eng Sci. 1988;43(1):91-102.

44. Boddington T, Gray P, Harvey DI. Thermal Theory of Spontaneous Ignition: Criticality in Bodies of Arbitrary Shape. Philos Trans $R$ Soc A Math Phys Eng Sci. 1971;270(1207):467-506.

45. Kang TS, Park $\mathrm{CH}$, Kim SH. Characteristics of exothermic reaction fronts in the gasless combustion system. Ceram Int. 2011;37(3):825-833.

46. Hwang S, Mukasyan AS, Varma A. Mechanisms of combustion wave propagation in heterogeneous reaction systems. Combust Flame. 1998;115(3):354-363.

47. Fiedler T, Belova IV, Broxtermann S, Murch GE. A thermal analysis on selfpropagating high temperature synthesis in joining technology. Comput Mater Sci. 2012;53(1):251-257.

48. Sánchez-Rodríguez D, Wada H, Yamaguchi S, Farjas J, Yahiro H. Synthesis of LaFeO3 perovskite-type oxide via solid-state combustion of a cyano complex precursor: The effect of oxygen diffusion. Ceram Int. 2017;43(3):3156-3165.

49. Mukasyan AS, Rogachev AS. Discrete reaction waves: Gasless combustion of solid powder mixtures. Prog Energy Combust Sci. 2008;34(3):377-416.

50. García E, Sánchez-Rodríguez D, López-Olmedo JP, Farjas J, Roura P. The effect of volatiles on the measurement of the reaction heat by differential scanning calorimetry. J Therm Anal Calorim. 2015;(121):187-194.

51. Enig JW. Approximate Solutions in the Theory of Thermal Explosions for Semi- 
Infinite Explosives. Proc R Soc A Math Phys Eng Sci. 1968;305(1481):205-217.

52. Akita K. Studies on the Mechanism of Ignition of Wood. Rep Fire Res Inst Japan. 1959;9(1-2):1-106.

53. Eloussifi H, Farjas J, Roura P, et al. Thermal decomposition of barium trifluoroacetate thin films. Thermochim Acta. 2013;556(0):58-62.

54. Khawam A, Flanagan DR. Basics and applications of solid-state kinetics: a pharmaceutical perspective. J Pharm Sci. 2006;95(3):472-498.

55. Boddington T, Hongtu F, Laye PG, Nawaz M, Nelson DC. Thermal runaway by thermal analysis. Thermochim Acta. 1990;170:81-87.

56. Sánchez-Rodríguez D, Wada H, Yamaguchi S, Farjas J, Yahiro H. Selfpropagating high-temperature synthesis of LaMO3 perovskite-type oxide using heteronuclearcyano metal complex precursors. J Alloys Compd. 2015;649:12911299.

57. Farjas J, Roura P. Isoconversional analysis of solid state transformations. A critical review. Part I. Single step transformations with constant activation energy. J Therm Anal Calorim. 2011;105(3):757-766.

58. Brown ME, Maciejewski M, Vyazovkin S, et al. Computational aspects of kinetic analysis: Part A: The ICTAC kinetics project-data, methods and results. Thermochim Acta. 2000;355(1-2):125-143.

59. Vyazovkin S, Burnham AK, Criado JM, Pérez-Maqueda LA, Popescu C, Sbirrazzuoli N. ICTAC Kinetics Committee recommendations for performing kinetic computations on thermal analysis data. Thermochim Acta. 2011;520(12):1-19.

60. Semenov N. Theories of combustion processes. Zeitschrift für Phys. 1928;48(78):571-582. 
61. Semenov N. Thermal Theory of Combustion and Explosion. Prog Phys Sci USSR. 1940;23(3):251-292.

62. Chambré PL. On the Solution of the Poisson-Boltzmann Equation with Application to the Theory of Thermal Explosions. $J$ Chem Phys. 1952;20(11):1795.

63. Gill W, Donaldson AB, Shouman AR. The Frank-Kamenetskii problem revisited. Part I. Boundary conditions of first kind. Combust Flame. 1979;36:217-232.

64. Harley C, Momoniat E. Alternate Derivation of the Critical Value of the FrankKamenetskii Parameter in Cylindrical Geometry. J Nonlinear Math Phys. 2008;15:69-76.

65. Farjas J, Rosell JI, Herrero R, Pons R, Pi F, Orriols G. Equivalent low-order model for a nonlinear diffusion equation. Phys $D$ Nonlinear Phenom. 1996;95(2):107-127.

66. Thomas PH. Some approximations in the theory of self-heating and thermal explosion. Trans Faraday Soc. 1960;56:833.

67. Casson V, Lister DG, Milazzo MF, Maschio G. Comparison of criteria for prediction of runaway reactions in the sulphuric acid catalyzed esterification of acetic anhydride and methanol. J Loss Prev Process Ind. 2012;25(1):209-217.

68. Kassoy DR, Poland J. The Subcritical Spatially Homogeneous Explosion; Initiation to Completion. Combust Sci Technol. 1975;11(3-4):147-152.

69. Casson V, Maschio G. Screening Analysis for Hazard Assessment of Peroxides Decomposition. Ind Eng Chem Res. 2012;51(22):7526-7535.

70. Thomas PH, Bowes PC. Some aspects of the self-heating and ignition of solid cellulosic materials. Br J Appl Phys. 1961;12(5):222-229.

71. Varma A, Morbidelli M, Wu H. Parametric Sensitivity in Chemical Systems (1st 
edition). New York: Cambridge University Press; 1999.

72. Bilous O, Amundson NR. Chemical reactor stability and sensitivity: II. Effect of parameters on sensitivity of empty tubular reactors. AIChE J. 1956;2(1):117-126.

73. Morbidelli M, Varma A. On parametric sensitivity and runaway criteria of pseudohomogeneous tubular reactors. Chem Eng Sci. 1985;40(11):2165-2168.

74. Frank-Kamenetskii DA. Nonstationary theory of thermal explosion. Zh Fiz Khim (J Phys Chem USSR). 1946;20(2):139-144.

75. Gray P, Lee PR. Thermal explosion theory. In: Tipper C, ed. Oxidation and Combustion Reviews, Vol 2. Amsterdam: Elsevier; 1967:1-184.

76. Ashby MF. Materials Selection in Mechanical Design. Vol 2. Oxford: Butterworth-Heinemann Limited; 1999.

77. Marchal W, De Dobbelaere C, Kesters J, et al. Combustion deposition of $\mathrm{MoO}_{3}$ films: from fundamentals to OPV applications. RSC Adv. 2015;5(111):9134991362.

78. Roura P, Farjas J, Eloussifi H, et al. Thermal analysis of metal organic precursors for functional oxide preparation: Thin films versus powders. Thermochim Acta. $2015 ; 601: 1-8$.

79. Eloussifi H, Farjas J, Roura P, et al. Thermoanalytical study of the decomposition of yttrium trifluoroacetate thin films. Thin Solid Films. 2013;545(0):200-204.

80. Kossoy AA, Sheinman IY. Comparative analysis of the methods for SADT determination. J Hazard Mater. 2007;142(3):626-638. 


\section{Figure captions}

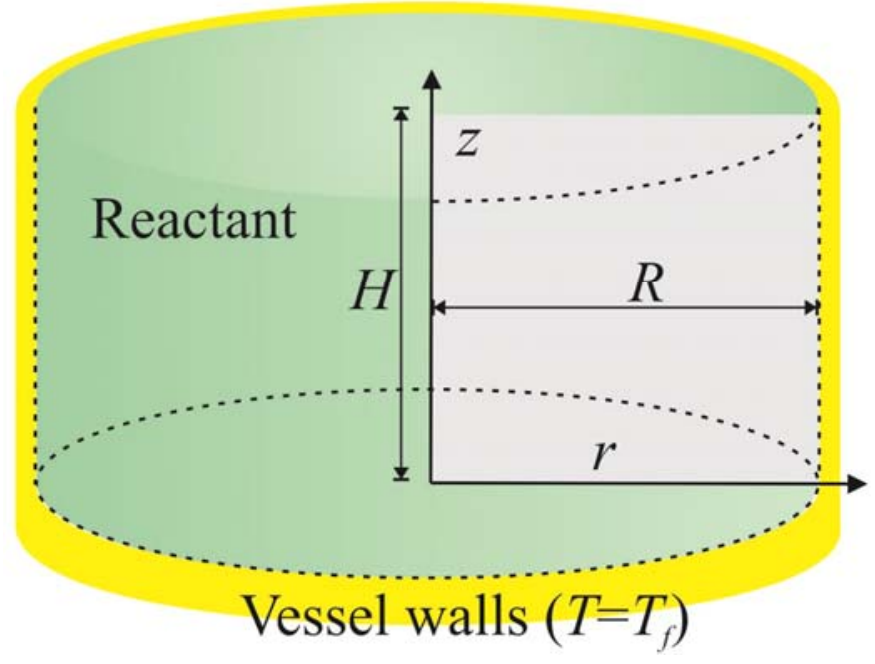

Figure 1. System geometry. The sample is placed inside a cylindrical vessel without a cover.

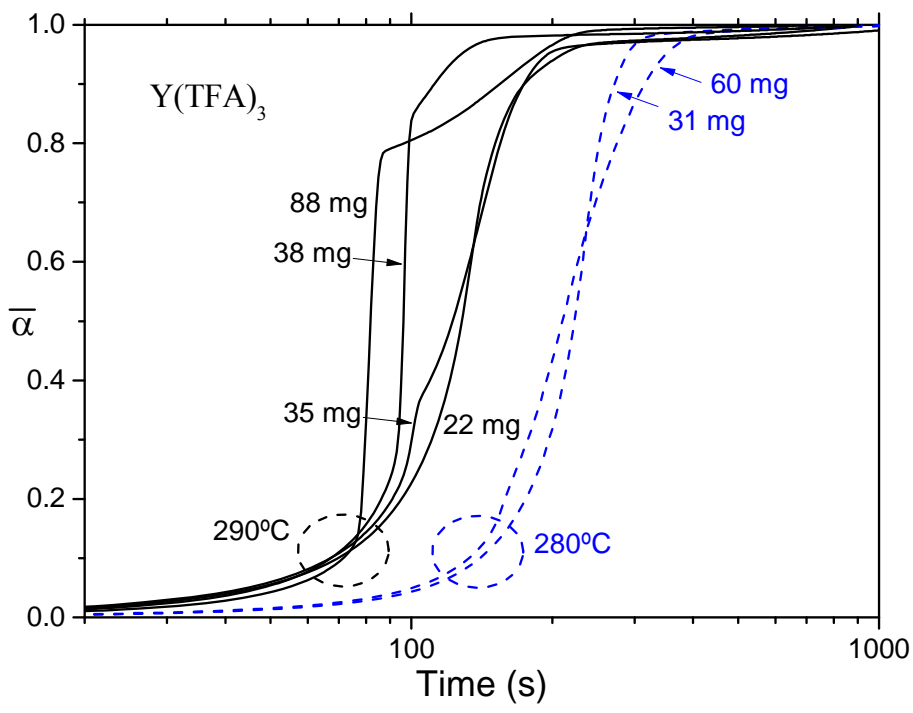

Figure 2. Thermal decomposition of $\mathrm{Y}(\mathrm{TFA})_{3}$ powders inside an alumina crucible for different initial sample masses. The furnace temperature is kept constant at 280 (dashed lines) and $290^{\circ} \mathrm{C}$ (solid lines). 


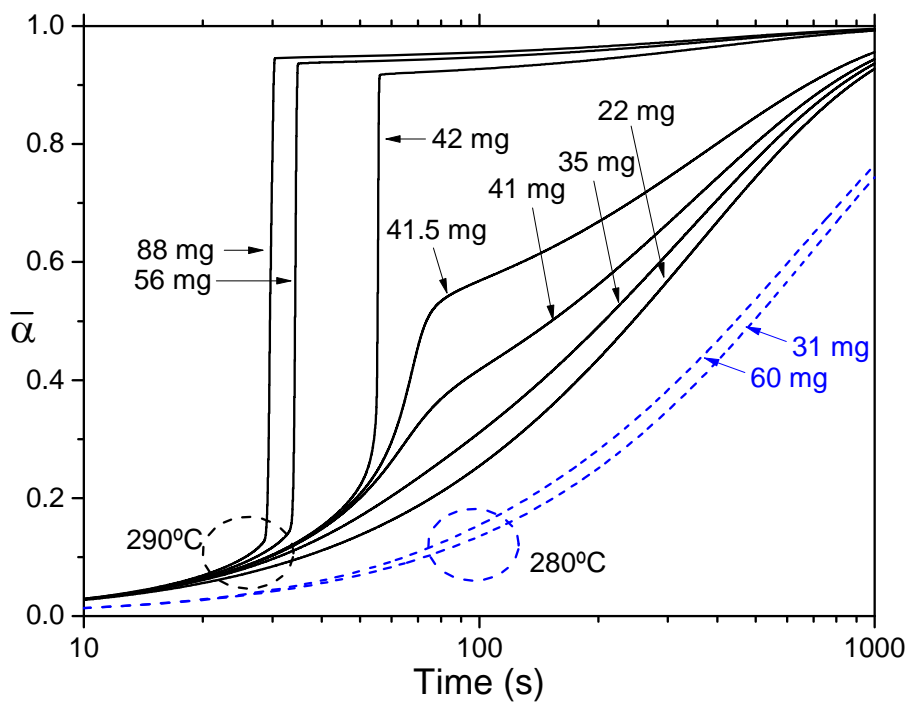

Figure 3. Numerical simulation of $\mathrm{Y}(\mathrm{TFA})_{3}$ thermal decomposition inside an alumina crucible. Simulation parameters of $\mathrm{Y}(\mathrm{TFA})_{3}$ powders are given in Table 1 . The internal radius of the crucible is 2.5 and the thickness of the crucible wall is $0.5 \mathrm{~mm}$. Thermal parameters of alumina are given in Table 2. The furnace temperature is set constant at 280 (dashed lines) and $290^{\circ} \mathrm{C}$ (solid lines).

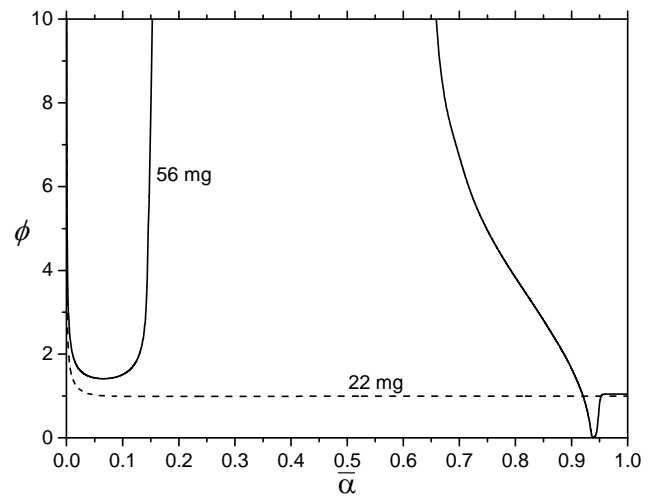

Figure 4. Evolution of the ratio between the heat generation and the heat dissipation rates, $\phi$, for an initial sample mass of $22 \mathrm{mg}$ (dashed line) and of $56 \mathrm{mg}$ (solid line) at $290^{\circ} \mathrm{C}$ (see Figure 3). 


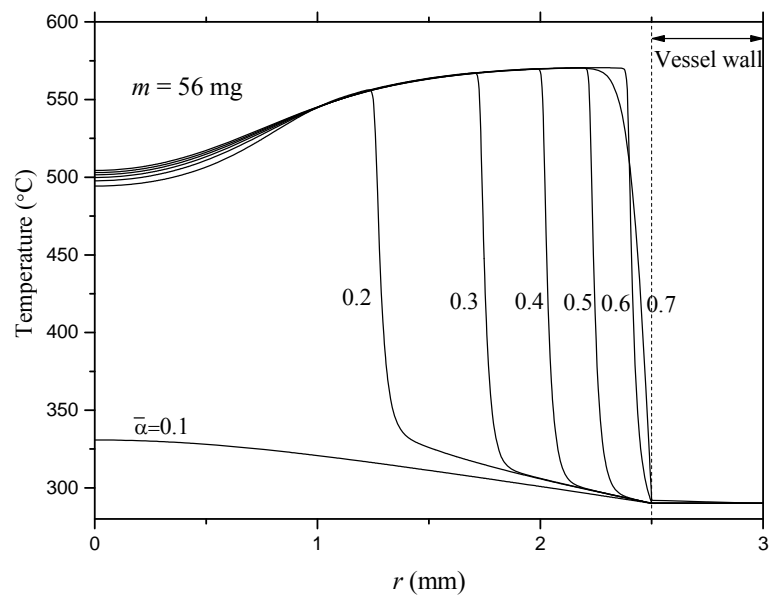

Figure 5. 1D numerical temperature profiles along the cylinder axis $(r=0)$ at different stages of $\mathrm{Y}(\mathrm{TFA})_{3}$ thermal decomposition. Simulation parameters coincide with those of Figure 3; sample mass $56 \mathrm{mg}$.

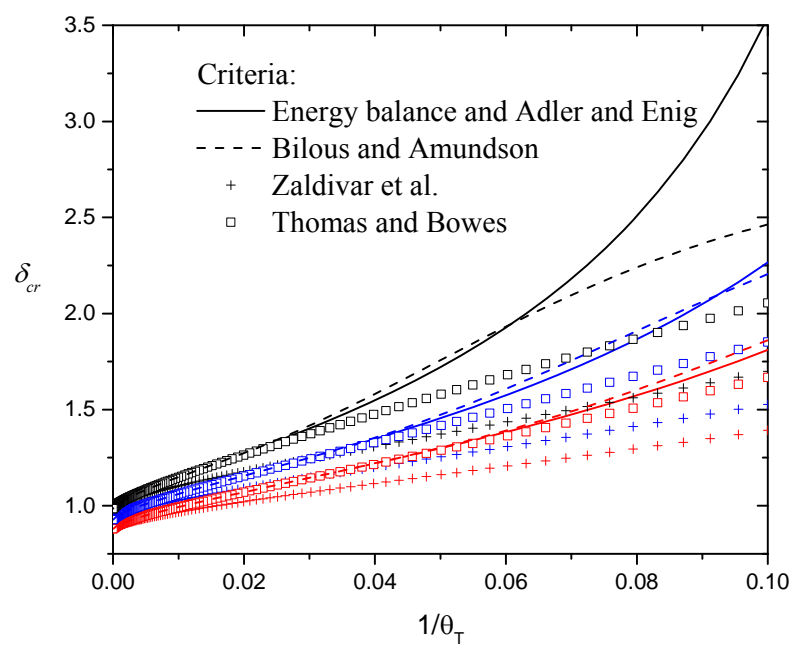

Figure 6. Lines are the value of $\delta_{c r}$ determined numerically the non-stationary model, Eq. (16) for different runaway criteria: energy balance (solid line), Bilous and Amundson (dashed line), Zaldivar et al. (crosses) and Thomas and Bowes (squares). Three values of $\varepsilon$ are analyzed (red $\varepsilon=0$, blue $\varepsilon=0.05$ and black $\varepsilon=1$ ) while $\theta_{T}$ is varied from 10 to $10^{5}$. 


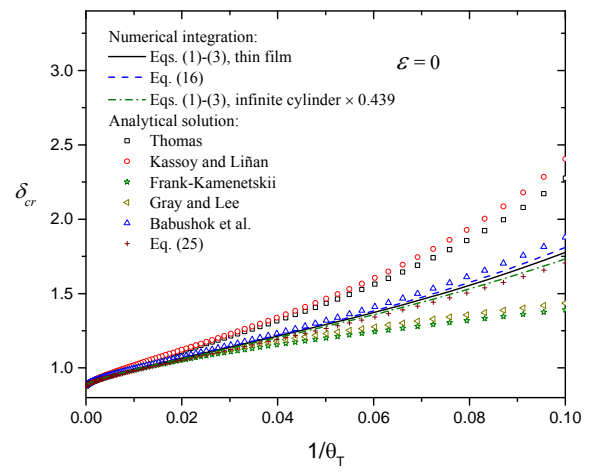

Figure 7. Lines are the value of $\delta_{c r}$ determined numerically from the exact model, Eqs. (1)-(3), and from the non-stationary model, Eq. (16). $\varepsilon$ is kept constant and equal to zero while $\theta_{T}$ is varied from 10 to $10^{5}$.Two different geometries have been analyzed: a thin film (or semi-infinite slab) and an infinite cylinder. For the sake of comparison the values of $\delta_{c r}$ for a cylinder has been multiplied by 0.439 . Symbols are the values of $\delta_{c r}$ determined from the critical conditions.

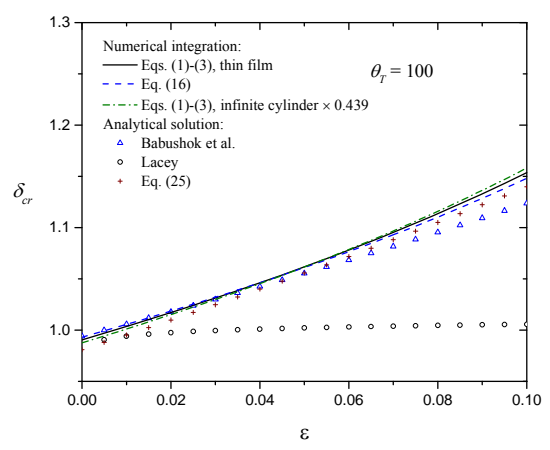

Figure 8. Lines are the value of $\delta_{c r}$ determined numerically from the exact model, Eqs. (1)-(3), and from the non-stationary model, Eq. (16). $\theta_{T}$ is kept constant and equal to 100.Two different geometries have been analyzed: a thin film (or semi-infinite slab) and an infinite cylinder. The value of $\delta_{c r}$ for a cylinder has been multiplied by 0.439 . Symbols are the value of $\delta_{c r}$ determined from the analytical solution of Babushok et al, Lacey and our critical condition. 


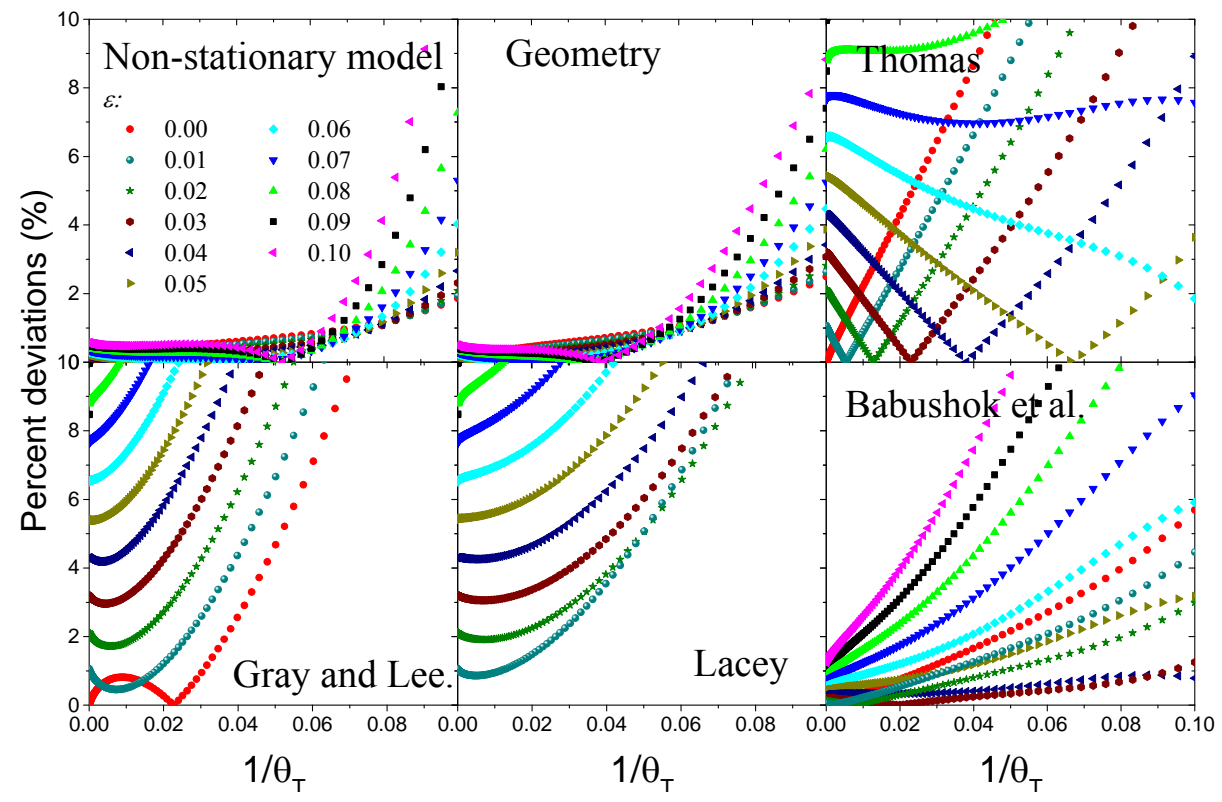

Figure 9. Percent deviations of $\delta_{c r}$ values determined from the non-stationary model and several runaway conditions with respect the exact solution. Also the deviations in $\delta_{c r}$ determined from the thin-film and infinite cylinder geometry are shown (The value of $\delta_{c r}$ for a cylinder has been multiplied by $\left.C_{T F}^{(1 D)} / C_{C y l}^{(1 D)}\right)$.

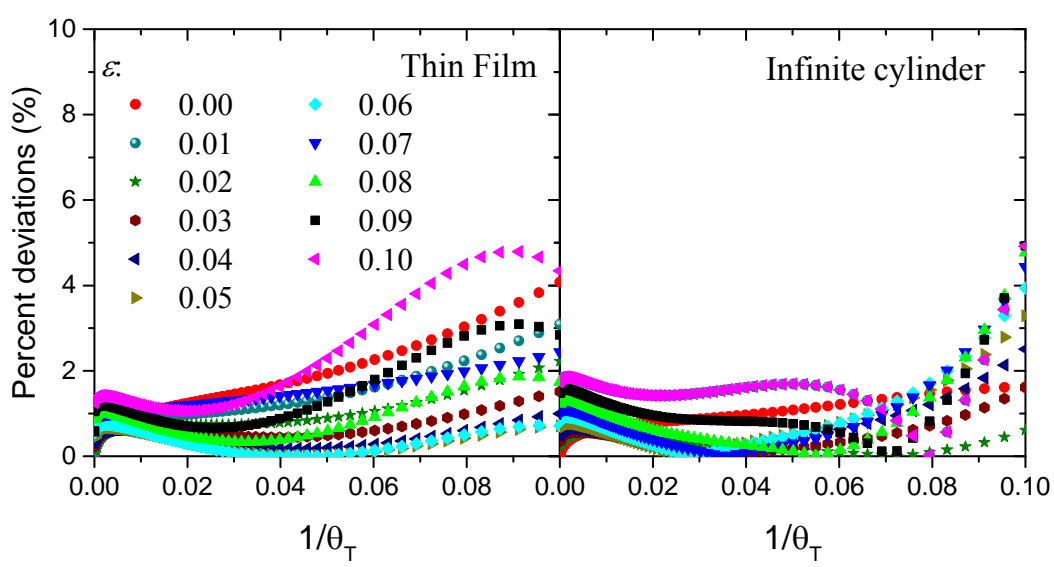

Figure 10. Percent deviations of $\delta_{c r}$ values determined from our runaway condition, Eq. (25) with respect the exact solution for a thin-film and an infinite cylinder geometries. 


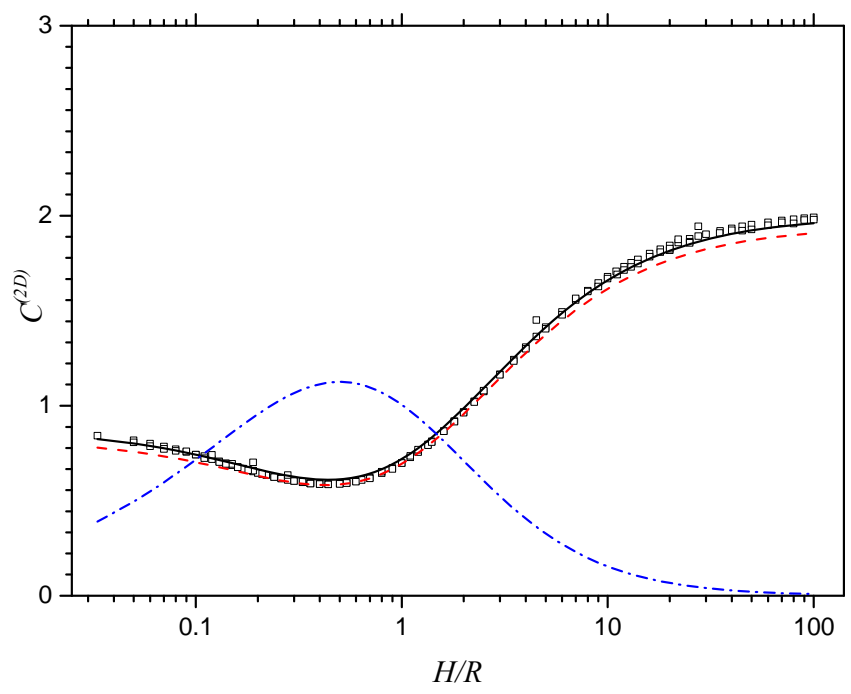

Figure 11. Symbols: parameter $C^{(2 D)}$ determined from Eq. (25). The values of $\delta, \varepsilon$ and $\theta_{\mathrm{T}}$ at the threshold of thermal runaway have been calculated numerically from the exact system, Eqs. (1)-(3). The solid line represents the values of $C^{(2 D)}$ determined from Eq. (31). The dash-dotted blue line corresponds to the value of $C^{(2 D)}$ obtained assuming a sphere of the same volume and the dashed red line has been derived using Kondratiev approximation. 


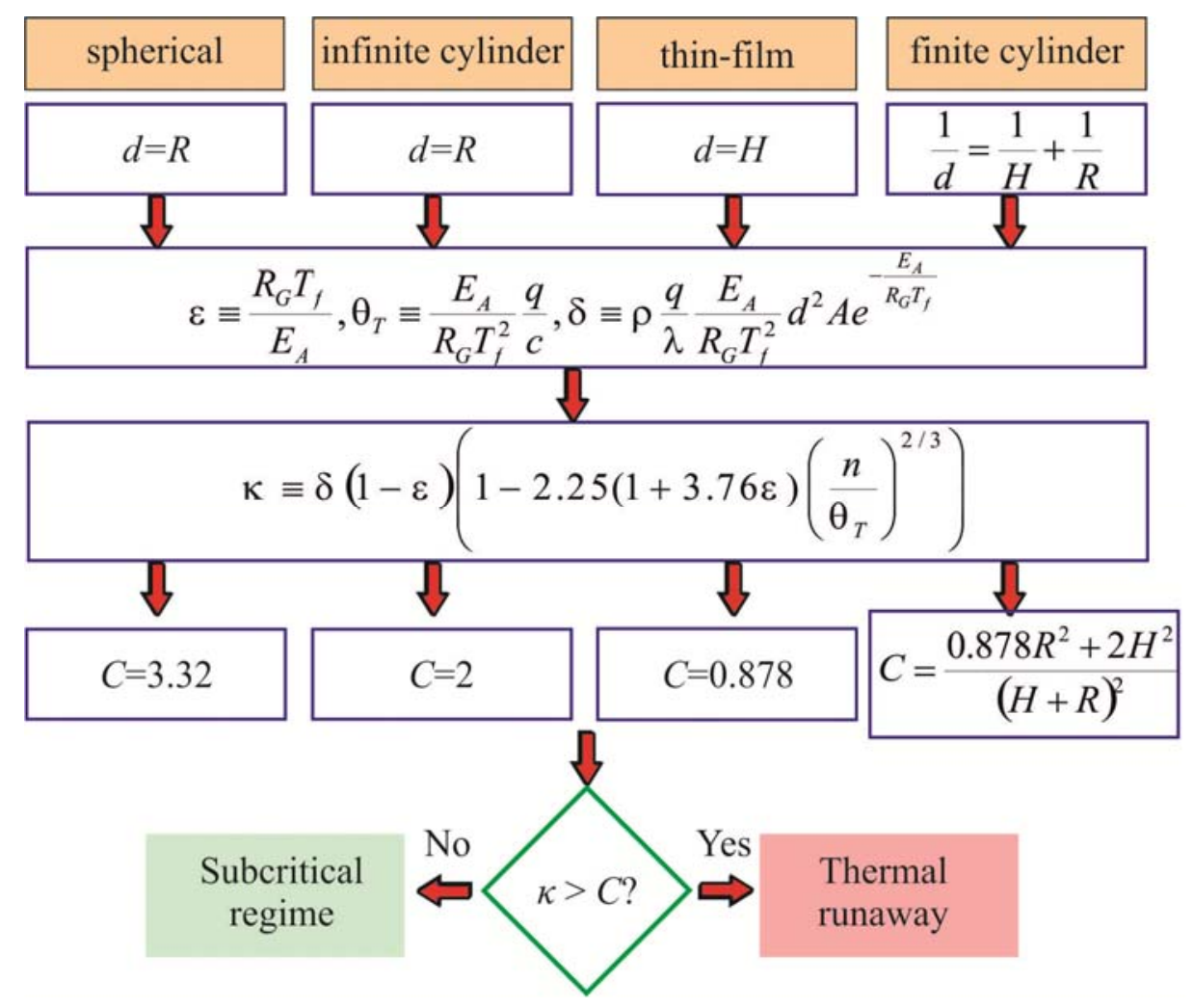

Figure 12. Steps to determine the occurrence of a thermal runaway.

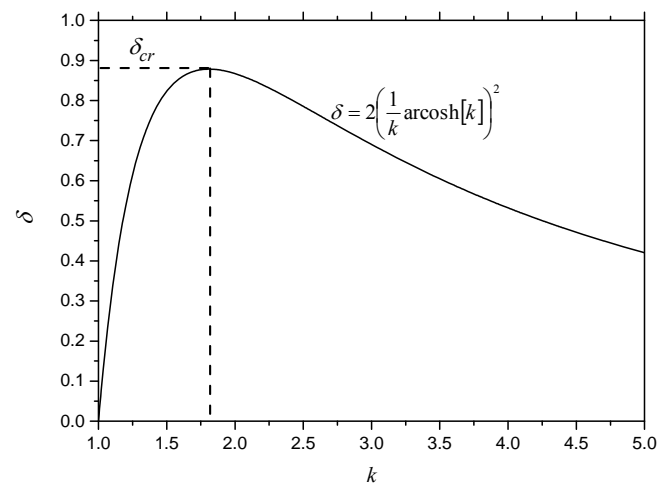

Figure A.1. Frank-Kamenetskii parameter as a function of the integration constant, Eq. (A.3). 


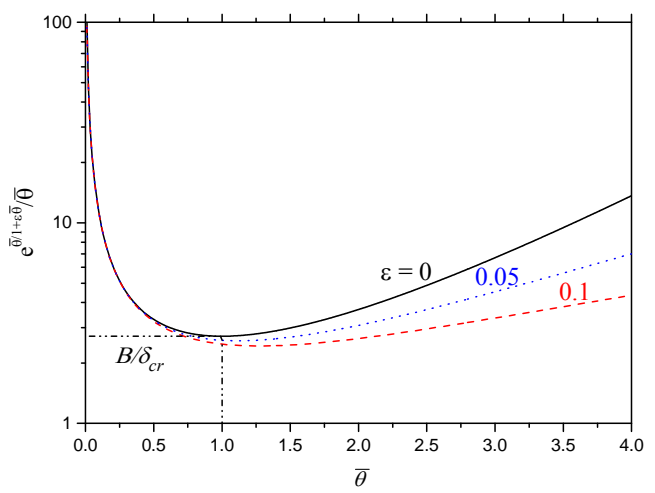

Figure B.1. Plot of $e^{\bar{\theta} / 1+\varepsilon \bar{\theta}} / \bar{\theta}$ for different values of $\varepsilon$.

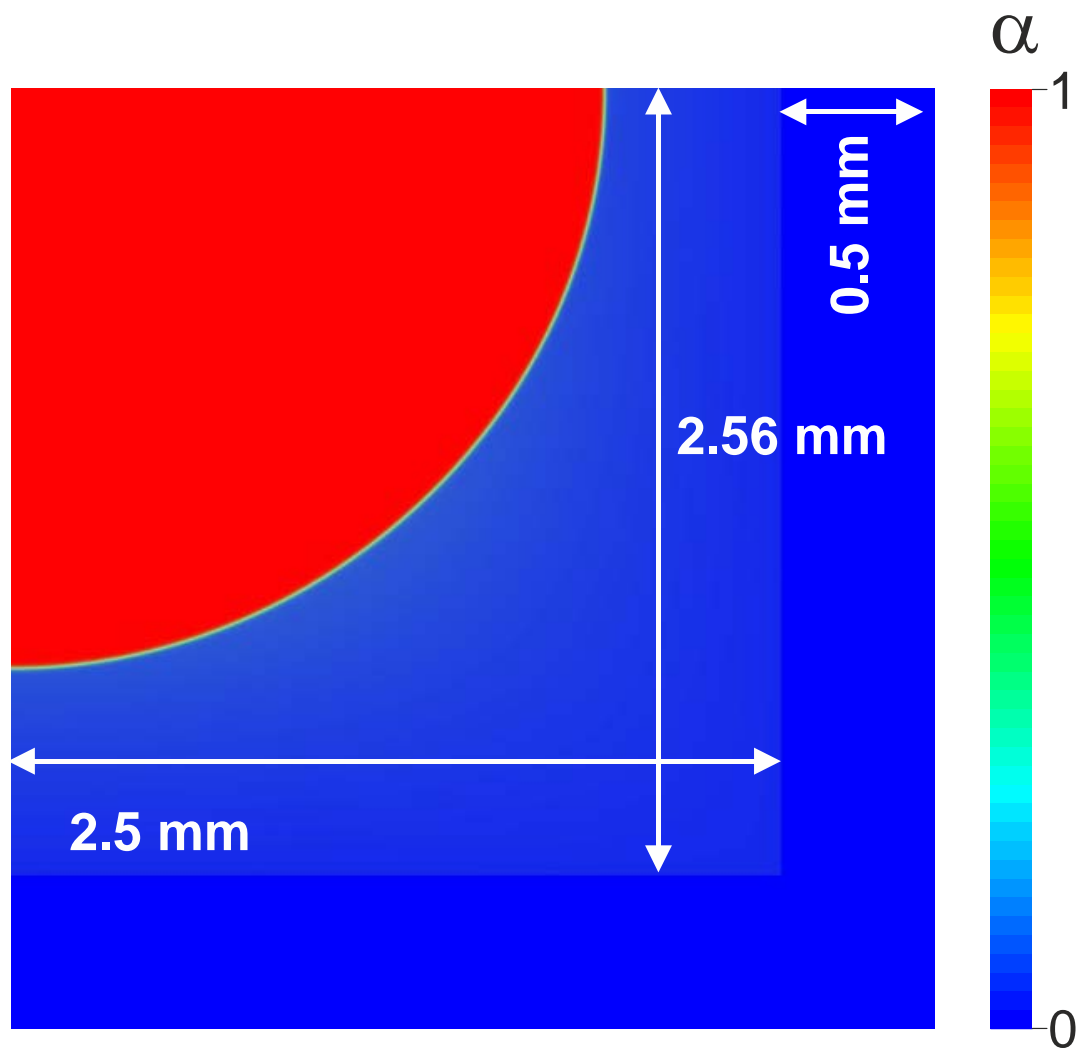

Movie 1. Snapshot of the evolution of the reaction for $\mathrm{Y}(\mathrm{TFA})_{3}$ powders inside an alumina crucible. The physical parameters are those indicated in Fig. 5. The movie is in the supplementary data. 
Table 1. Physical parameters of Y(TFA $)_{3}$.

\begin{tabular}{ll}
\hline Thermal conductivity, $\lambda, \mathrm{W} /(\mathrm{m} \cdot \mathrm{K})$ & 0.06 \\
Specific heat capacity, $c, \mathrm{~J} /(\mathrm{kg} \cdot \mathrm{K})$ & 875 \\
Density, $\rho, \mathrm{kg} / \mathrm{m}^{3}$ & 1114 \\
Specific heat of reaction, $q, \mathrm{~J} / \mathrm{kg}$ & $2.75 \times 10^{5}$ \\
Activation energy, $E_{A}, \mathrm{~J} / \mathrm{mol}$ & $1.74 \times 10^{5}$ \\
Pre-exponential constant, $A, \mathrm{~s}^{-1}$ & $3.4 \times 10^{13}$ \\
\hline
\end{tabular}


Table 2. Physical parameters of $\mathrm{Al}_{2} \mathrm{O}_{3}$.

\begin{tabular}{ll}
\hline Thermal conductivity, $\lambda, \mathrm{W} /(\mathrm{m} \cdot \mathrm{K})$ & 39 \\
Specific heat capacity, $c, \mathrm{~J} /(\mathrm{kg} \cdot \mathrm{K})$ & 775 \\
Density, $\rho, \mathrm{kg} / \mathrm{m}^{3}$ & 3980 \\
Thermal diffusivity, $\mathrm{m}^{2} / \mathrm{s}$ & $1.3 \times 10^{-5}$ \\
\hline
\end{tabular}

